\title{
Fault Tolerant Position-mooring Control for Offshore Vessels
}

\author{
Blanke, Mogens; Nguyen, Trong Dong
}

Published in:

Ocean Engineering

Link to article, DOI:

10.1016/j.oceaneng.2017.11.042

Publication date:

2018

Document Version

Peer reviewed version

Link back to DTU Orbit

Citation (APA):

Blanke, M., \& Nguyen, T. D. (2018). Fault Tolerant Position-mooring Control for Offshore Vessels. Ocean Engineering, 148, 426-441. https://doi.org/10.1016/j.oceaneng.2017.11.042

\section{General rights}

Copyright and moral rights for the publications made accessible in the public portal are retained by the authors and/or other copyright owners and it is a condition of accessing publications that users recognise and abide by the legal requirements associated with these rights.

- Users may download and print one copy of any publication from the public portal for the purpose of private study or research.

- You may not further distribute the material or use it for any profit-making activity or commercial gain

- You may freely distribute the URL identifying the publication in the public portal

If you believe that this document breaches copyright please contact us providing details, and we will remove access to the work immediately and investigate your claim. 


\title{
Fault Tolerant Position-mooring Control for Offshore Vessels
}

\author{
Mogens Blanke ${ }^{\mathrm{a}, *}$, Dong T. Nguyen ${ }^{\mathrm{b}}$ \\ ${ }^{a}$ AMOS Centre of Excellence, Institute for Technical Cybernetics, Norwegian University of \\ Science and Technology, NO 7491, Trondheim, Norway, and \\ Department of Electrical Engineering, Technical University of Denmark, DK 2800, Kongens \\ Lyngby, Denmark \\ ${ }^{b}$ CeSOS Centre of Excellence, Department of Marine Technology, Norwegian University of \\ Science and Technology, NO 7491, Trondheim, Norway, now \\ DNV-GL, Vestre Rosten 77, Tiller, Norway.
}

\begin{abstract}
Fault-tolerance is crucial to maintain safety in offshore operations. The objective of this paper is to show how systematic analysis and design of fault-tolerance is conducted for a complex automation system, exemplified by thruster assisted Position-mooring. Using redundancy as required by classification societies' class notations for offshore position controlled vessels, the paper shows how violations of normal behaviour of main components can be detected and isolated. Using a functional service philosophy, diagnosis procedures are auto-generated based on provable correct graph analysis methods. Functional faults that are only detectable, are rendered isolable through an active isolation approach. Once functional faults are isolated, they are handled by fault accommodation techniques to meet overall control objectives specified by class requirements. The paper illustrates the generic methodology by a system to handle faults in mooring lines, sensors or thrusters. Simulations and model basin experiments are carried out to validate the concept for scenarios with single or multiple faults. The results demonstrate that enhanced availability and safety are obtainable with this design approach. While methods are introduced at a tutorial level, the paper is original by providing a total Position-mooring system design that ensures resilience to any single fault and to selected multiple faults.
\end{abstract}

Keywords: Safety, fault-tolerant control, FPSO, Position-mooring, active fault isolation, fault diagnosis

\footnotetext{
${ }^{*}$ Corresponding author

Email addresses: mb@elektro.dtu.dk (Mogens Blanke), dong.nguyen@dnvgl.com (Dong
} T. Nguyen) 


\section{Introduction}

Safety and cost effectiveness are primary concerns for positioning control systems for marine vessels Gray and Macdonald (1982); Chen et al. (2009). Frequent shutdowns of the whole control system when simple faults occur are costly and high risk events for humans, for equipment and for environment.

The present approach to fault handling in the marine industry is to call for human intervention when faults occur. The study of Chen and Moan (2004) showed that when faults occur in a positioning control system performing tandem operations, the time window for the operator is less than two minutes to avoid collision. With this time to react, human intervention is likely to fail to recover the system and autonomous handling of faults could enhance safety and availability of offshore operations. Some PM system designs have the ability to handle selected faults of particular high severity, but these designs have been made ad-hoc without a uniform approach to fault handling. Ad-hoc implemented fault handling increases the risk of software faults that could become critical as this part of code is only executed when some failure has happened and handling of a not-normal situation is needed. Tools for systematic analysis and design for fault tolerant control (FTC) are therefore adopted in this paper and used to suggest a complete FTC design and analysis procedure for a thruster assisted Position-mooring system.

Means toward systematic design for fault-tolerant control have been available and have been used for individual subsystems or functions on marine vessels. Fault diagnosis of a diesel-driven propulsion system was the subject of the benchmark in Izadi-Zamanabadi and Blanke (1999), and solutions to the diagnosis part of the problem demonstrated by a sliding mode observer in Edwards and Spurgeon (2000), an adaptive observer in Blanke et al. (1998), and a nonswitching detection and accommodation solution in Wu et al. (2006). Larger subsystems were treated in Blanke (2005), for station keeping control, where the structure-graph approach was successfully employed, and fault-tolerant sensor fusion for navigation instruments was treated in Blanke (2006). For Positionmooring, a setpoint chasing control algorithm was made fault-tolerant in Fang and Blanke (2011) and reliability indexes were included in the optimization in Fang et al. (2013). The minimum thruster power position was calculated in Wang et al. (2016), without considering faults or fault-tolerance. Reliability indexes were also considered in Wang et al. (2014) who suggested a backstepping control approach for Position-mooring, but they did not consider faults or fault-tolerance. The earlier reported results, which treated not-normal conditions, looked at selected faults in mooring lines and in Blanke et al. (2012), also on buoyancy element failures. In these earlier studies, fault isolation was made possible by assuming certain parts of the PM system to be healthy. This study will include the possibility that one or more sensors or actuators are faulty and it will include disturbances from sea, wind and current. This makes fault isolation an issue of special concern. The present problem is hence significantly more complex, yet also more realistic than earlier research. The study is made generic and realistic to the maritime industry through considering the instrumentation 
that is required by classification societies.

With the objective of presenting a systematic FTC design that can be conducted already at the design stage of a vessel automation system, with limited additional efforts, structural analysis is employed for analysis of fault detection and isolation properties, considering single or multiple-faults. Using standard cases of instrumentation, as advised by classification societies, it is shown that fault isolation cannot always be obtained with traditional methods, but an active fault isolation technique is then explored with the purpose of isolating faults that are otherwise only detectable. The paper details on how this is done and it shows how, once a fault is isolated, it can be handled fast and predictably without human intervention. The implications for marine operations are emphasised and aspects of overall safety and availability are considered. The suggested techniques are implemented and tested in a model basin demonstrating the suggested approach in a fully autonomous Position-mooring system.

The paper is organised as follows. After an overview of prior research in the area, Section 3 introduces the modelling at a control plant level for FTC purposes. Section 4 presents fault diagnosis for fault detection and isolation using structural analysis and extends these with active isolation techniques. Section 5 shows design of controllers to handle the range of faults dealt with and the proposed fault-tolerant DP system design is validated by model basin tests in Section 6. Section 7 concludes the paper. Notations and abbreviations are listed in Tables 1 and 2.

\section{Background and Previous Research}

In offshore operations, marine vessels are often required to be kept in a desired position using dynamic positioning (DP) or Position-mooring (PM) system. The term positioning control is commonly used to denote either of these technologies. A DP system exclusively uses thrusters to achieve a desired position and heading. Research on this industrially important subject include Balchen et al. (1980), Selkäinaho (1993), IMO (1994), Sørensen et al. (1996), Strand et al. (1998), Strand (1999), Sørensen and Strand (2000), Fossen (2002), Lindegaard (2003), Sørensen (2005), and Tannuri and Morishita (2006). The historiy of DP development was excellently described in Breivik et al. (2015). The vigor of the area is evidenced by new ideas being presented to dynamic positioning control, e.g. Hassani et al. (2017), Benetazzo et al. (2015), Wu et al. (2016), use of position estimation techniques when anchor positions are uncertain Ren and Skjetne (2016) and study of key performance indicators in Park et al. (2016). Fault-tolerant control for non-moored vessels were presented in Blanke (2005), Benetazzo et al. (2015). Classification society rules for the area are found in DNV (2014).

Position-moored systems use a combination of mooring lines and thrusters to maintain the vessel's position, balancing the mean ocean disturbances acting on the vessel. External forces are mainly attenuated by the mooring system while the thrusters are used as dampers to reduce the vessel's dynamical motions. In harsh weather conditions, the use of thrusters is necessary in a PM system 


\begin{tabular}{ll}
\hline $\mathbf{A}_{b t}$ & thruster force to body force-moment matrix \\
$\mathbf{A}_{b m}$ & mooring line force to body force-moment matrix \\
$\mathbf{D}$ & linear damping matrix \\
$\boldsymbol{\eta}=[\mathbf{p} \top, \psi]^{\top}$ & position and heading \\
$F_{i}$ & thrust from $i^{t h}$ thruster \\
$\mathbf{h}=[z, \phi, \theta]^{\top}$ & heave, roll and pitch of the vessel \\
$\mathbf{M}$ & body mass matrix including hydrodynamic added mass \\
$N_{m}, N_{t}, N_{p}$ & number of mooring lines, thrusters and position sensors \\
$N_{g}, N_{v}, N_{w}$ & number of horizontal -, vertical gyroscopes and anemometers \\
$\boldsymbol{\nu}=[u, v, r]^{\top}$ & velocity vector of the vessel in the body-fixed frame \\
$\boldsymbol{\omega}=[p, q, r]^{\top}$ & angular velocity of body \\
$\mathbf{p}=[x, y]^{\top}$ & North-East position vector in Earth-fixed frame \\
$\psi, r$ & yaw angle and yaw rate of the vessel \\
$\mathbf{R}_{n b}(\psi)$ & yaw rotation from body to navigation frame \\
$T_{j}, T_{j}^{x y}$ & tension in $j$ th mooring line and its horizontal component \\
$u_{i}$ & command shaft speed to $i^{t h}$ thruster \\
$\mathbf{v}_{\mathrm{w}}, \mathbf{v}_{\mathrm{c}}$ & wind and current velocity vectors \\
\hline
\end{tabular}

Table 2: Acronyms and Abbreviations.

\begin{tabular}{ll} 
ARR & analytical redundancy relation \\
AUTS, AUT, AUTR & DNV-GL class notations for DP \\
CUSUM & cumulative sum \\
DP & dynamic positioning \\
FTC & fault-tolerant control \\
FPSO & floating production storage and offloading \\
GPS & global positioning system \\
HPR & hydro-acoustic position reference \\
MSO & minimial structurally overdetermined \\
PM & Position-mooring with thruster assistance \\
RPM & revolutions per minute \\
\hline
\end{tabular}


in order to avoid large tensions in mooring lines and to provide compensation of any line break by keeping the vessel at an appropriate setpoint. Switching strategies with a bank of controllers to cope with weather conditions were in particular studied in Nguyen et al. (2007). Setpoint chasing control was suggested in Sørensen et al. (2001) and extended in Nguyen and Sørensen (2009b). Using a mooring line reliability criterion as control objective, Berntsen et al. (2008) showed how to prevent line overload. A semi-static setpoint recalculation approach was suggested in Fang and Blanke (2011) to deal with breakage of one or more lines and protect remaining intact ones. Selected faults, line breakage and loss of an underwater buoyancy element, were demonstrated to be diagnosable in a position-moored system by Fang et al. (2015). This paper considers the much wider scenario of the entire fault-tolerant control problem including failure of any of the key instruments, actuators or mooring lines. Active fault diagnosis techniques Niemann (2006), Blanke and Staroswiecki (2006), Poulsen and Niemann (2008), Gelso and Blanke (2009) and Niemann (2012) are designed and tested in this paper to isolate faults that are only detectable with conventional diagnostic methods.

Being an area of significant industrial importance, fault prognosis and diagnosis methods have been studied extensively. Methods employing spectral and time-domain properties are extremely useful for diagnosis in rotating machinery Feng. et al. (2013) and Sun et al. (2014) whereas model-based diagnosis has been the main focus for process diagnosis. A method that has proven to be useful for analysis of rather complex systems is structural analysis where graph-based tools are used to analyse topology while avoiding very detailed modelling. The theoretical foundation was provided in Dulmage and Mendelsohn (1959) and brought to use in fault diagnosis in Staroswiecki and Declerck (1989), Blanke et al. (2015) and Travé-Massuyès et al. (2006). The structure graph of a system helps discover potential redundancies and shows how to reconfigure the system when a component is disabled, e.g. due to faults. A hypothesis test mechanism, see Kay (1998), Basseville and Nikiforov (2002), and Travé-Massuyès (2014) evaluates whether the system behaviour resembles a faultless or faulty plant model. Successful application have been reported in several studies, see Noura et al. (2009) and in particular the automotive industry has been very active in this area, see Svärd et al. (2014). Once a fault is isolated to be in a specific component of the system, changes need to be made achieve given control objective, perhaps with degraded performance. Further details on FTC can be found in Blanke et al. (2015).

\section{Modelling for fault-tolerant control}

Modelling of marine systems is based on first principles with addition of laws from hydrodynamics. With a vessel being a body moving in six degrees of freedom, nonlinear static and dynamic phenomena are present. Hence, models will be nonlinear by nature and they easily become extremely complex. When aiming at analysis of features essential for fault detectability and for fault-tolerant control, the very detailed models pose an obstacle rather than a benefit because 
a first concern in fault-tolerant design is to gain insight in properties at an overall level of functionality. The modelling needed for fault-tolerant design is the topology of the system and descriptions of function blocks. This is done through formulation of constraints.

\subsection{Using constraints for modelling}

When considering fault-tolerant control, we need an answer to the question: which overall functions (e.g. actuators and sensors) are healthy and available for use by the control system. We are not interested in localization of defects to particular sub-components, as is the case for condition monitoring and maintenance systems. Modelling therefore need be done at the level of overall functionality. Such modelling is conveniently done using the principles of behavioural modelling Willems (1996), where constraints $c$ describe how certain variables are related. To introduce the notation, let variables be $x, z$ and $u$, and let $g_{s}$ and $g_{d}$ denote functions; then constrains can be static $\left(c_{s}\right)$ or dynamic $\left(c_{d}\right)$ :

$$
\begin{aligned}
& c_{s}: \quad 0=g_{s}(x, u)-z \\
& c_{d}: \quad 0=g_{d}(x, u)-\dot{x},
\end{aligned}
$$

where $g_{s}$ and $g_{d}$ can be linear or nonlinear. Derivatives of variables can be explicit or implicit in the constraints. Behavioural models are not necessarily continuous, but the framework of static and dynamic nonlinear constraints fit well with the physical modelling of a marine vessel.

While any symbol could be used for a constraint, we use $a_{i}$ for constraints related to actuators, $c_{i}$ for a generic constraints within the system, $m_{i}$ for measurement constraints and $d_{i}$ for differential constraints. The

\subsection{Model of a Position-moored marine vessel}

Consider a vessel with the thrusts produced by propellers,

$$
\mathrm{a}_{i}: F_{i}=g_{p}\left(u_{i}\right),
$$

where $i=1, \ldots, N_{\mathrm{t}} ; N_{\mathrm{t}}$ is the number of thrusters; $u_{i}$ is demanded propeller speed; $g_{p}$ is the nominal relation between $u_{i}$ and the thrust obtained. The thrusters contribute to control forces in surge and sway, and moment in yaw (hereinafter called control forces) according to their position in the vessel and azimuth (relative direction) of the thruster.

For brevity, we define the posture $\boldsymbol{\eta}$ as the vector of the Earth-fixed position of centre of the ship, $\mathbf{p} \in \mathbb{R}^{2}$, and its' heading angle, $\psi \in \mathbb{S}$,

$$
\boldsymbol{\eta}=\left[\mathbf{p}^{\top}, \psi\right]^{\top} .
$$

Horizontal plane forces from the Position-mooring (PM) system are available via the horizontal components of the tensions in mooring lines and the mooring system geometry. Tension in line $j$ is a function of position of the turret, hence a function of the posture vector,

$$
\mathrm{c}_{j}: T_{j}^{x y}=g_{j}(\boldsymbol{\eta}),
$$


where $j=1, \ldots, N_{\mathrm{m}}$ and $N_{\mathrm{m}}$ is the number of mooring lines. This constraint is the elastic catenary equations Triantafyllou (1990) for each mooring line,

$$
\begin{aligned}
x_{1}= & \frac{T_{i}^{x y}}{w_{0}} \sinh ^{-1}\left[\frac{T_{i}^{z}-w_{0}(L-s)}{T_{i}^{x y}}\right] \\
& -\frac{T_{i}^{x y}}{w_{0}} \sinh ^{-1}\left[\frac{T_{i}^{z}-w_{0} L}{T_{i}^{x y}}\right]+\frac{T_{i}^{x y} s}{E A_{0}} \\
x_{2}= & \frac{T_{i}^{x y}}{w_{0}} \sqrt{1+\left[\frac{T_{i}^{z}-w_{0}(L-s)}{T_{i}^{x y}}\right]^{2}} \\
& -\frac{T_{i}^{x y}}{w_{0}} \sqrt{1+\left[\frac{T_{i}^{z}-w_{0} L}{T_{i}^{x y}}\right]^{2}} \\
& +\frac{1}{E A_{0}}\left(T_{i}^{z} s+\frac{w_{0}}{2}\left[(L-s)^{2}-L^{2}\right]\right) .
\end{aligned}
$$

Here, $L$ is the un-stretched cable length; $s$ is a parameter running along the cable from 0 (anchor point) to $L$ (top end point); $x_{1}(s)$ and $x_{2}(s)$ are the spacial horizontal and vertical components along the cable; $T_{\mathrm{mo}}^{x y}$ and $T_{\mathrm{mo}}^{z}$ are the horizontal and vertical tensions at the upper end; $w_{0}$ is the weight in water per unit length; $E$ is Young's modulus of elasticity; and $A_{0}$ is the cross-sectional area. In order to calculate $T_{\mathrm{mo}}^{x y}$, we need to solve the catenary equation with boundary conditions, details of which are found in Aamo and Fossen (2001),

$$
\begin{aligned}
& x_{1}(0)=0, \quad x_{1}(L)=\quad \text { calculated from posture vector } \boldsymbol{\eta}, \\
& x_{2}(0)=0, \quad x_{2}(L)=\text { water depth. }
\end{aligned}
$$

We consider a rotatable turret mooring system in this paper. In this system, the ship can rotate freely around the turret; therefore, manual rotation of the turret is not considered.

Now consider the ocean current and wind disturbances acting on the vessel. The vector of sea current velocity over ground is $\mathbf{v}_{\mathrm{c}} \in \mathbb{R}^{2}$, relative wind is $\mathbf{v}_{\mathrm{w}} \in \mathbb{R}^{2}$, and $\boldsymbol{\nu} \in \mathbb{R}^{3}$ is a vector with body-fixed velocities relative to water in surge, sway and yaw. Roll, pitch and heave are not relevant for a moored vessel. Wind load is described by a function $\mathbf{g}_{\mathrm{W}}\left(\mathbf{v}_{\mathrm{w}}\right)$. The kinetics of the vessel in surge, sway and yaw is then,

$$
\begin{aligned}
\mathrm{c}_{1+N_{\mathrm{m}}} & : \mathbf{M} \dot{\boldsymbol{\nu}}=\sum_{i=1}^{N_{\mathrm{t}}} \mathbf{A}_{b t}^{i} F_{i} \\
+ & \sum_{j=1}^{N_{\mathrm{m}}} \mathbf{A}_{\mathrm{mo}}^{j}(\boldsymbol{\eta}) T_{j}^{x y}+\mathbf{g}_{\mathrm{W}}\left(\mathbf{v}_{\mathrm{w}}\right)-\mathbf{D}(\boldsymbol{\nu}) \boldsymbol{\nu}
\end{aligned}
$$

where $\mathbf{A}_{b t}$ is the mapping from individual thrusts to control forces in body coordinates, $\mathbf{A}_{\text {mo }}^{j}$ transforms the horizontal tension of the $j^{\text {th }}$ mooring line to body-fixed coordinates, and $\mathbf{D}(\boldsymbol{\nu}) \boldsymbol{\nu}$ is velocity-proportional damping. This term 
describes the combination of viscous drag on hull and mooring lines. In the sequel, we use $\mathbf{D} \boldsymbol{\nu}$ for brevity for this damping term.In reality, parts of the mooring lines have velocity through water that deviate from $\boldsymbol{\nu}$, e.g. due to vibrations. Such dynamic effects constitute, together with other nonlinear hydrodynamic phenomena and parameter uncertainties, so-called unmodelled dynamics. Fault diagnosis methods are designed such that they minimize sensitivity to unknown disturbances and to unknown dynamics.

The kinematics from body-fixed velocity relative to water, $\boldsymbol{\nu} \in \mathbb{R}^{3}$, to Earthfixed position and angles, $\boldsymbol{\eta} \in \mathbb{R}^{3}$, is

$$
\mathrm{c}_{2+N_{\mathrm{m}}}: \dot{\boldsymbol{\eta}}=\mathbf{R}_{n b}(\psi) \boldsymbol{\nu}+\left[\mathbf{v}_{\mathrm{c}}^{\top}, 0\right]^{\top}
$$

where $\mathbf{R}_{n b}(\psi)$ is the horizontal rotation from body to North-East coordinates, using the approximation that velocities are considered to be horizontal.

Using derivatives explicitly in the constraints, the differential operator need be described as a relation between a variable and its time derivative,

$$
\begin{aligned}
& \mathrm{d}_{1}: \quad \dot{\boldsymbol{\nu}}=\frac{d}{d t} \boldsymbol{\nu}, \\
& \mathrm{d}_{2}: \quad \dot{\boldsymbol{\eta}}=\frac{d}{d t} \boldsymbol{\eta} .
\end{aligned}
$$

\subsection{Sensors available}

The sensor devices onboard are: $N_{g}$ gyrocompass units for heading measurements; $N_{p}$ position measurements: GPS receivers and/or hydro-acoustic position reference (HPR) units; $N_{r}$ motion reference units (MRU) measuring heave, roll and pitch; $N_{w}$ anemometers for wind speed and direction measurements; a tension sensor for each of $N_{m}$ mooring lines; $N_{t}$ thrusters. The constraints that map the measured variables to the physical system states are,

$$
\begin{array}{rll}
\mathrm{m}_{k} & : h_{k}=\psi \\
\mathrm{m}_{N_{\mathrm{g}}+l} & : \mathbf{p}_{\mathrm{m}_{l}}=\mathbf{p}+\mathbf{R}(\phi, \theta, \psi) \mathbf{l}_{l} \\
\mathrm{~m}_{N_{\mathrm{g}}+N_{\mathrm{p}}+m} & : \mathbf{h}_{m_{m}}=[z, \phi, \theta]^{\mathrm{T}} \\
\mathrm{m}_{N_{\mathrm{g}}+N_{\mathrm{p}}+N_{\mathrm{r}}+n} & : \mathbf{w}_{\mathrm{m}_{n}}=\mathbf{v}_{\mathrm{w}} \\
\mathrm{m}_{N_{\mathrm{g}}+N_{\mathrm{p}}+N_{\mathrm{r}}+N_{\mathrm{w}}+1} & : \mathbf{c}_{m}=\mathbf{v}_{\mathrm{c}} \\
\mathrm{m}_{N_{\mathrm{g}}+\ldots+N_{\mathrm{w}}+1+i} & : u_{\mathrm{m}_{i}}=u_{i} \\
\mathrm{~m}_{N_{\mathrm{g}}+\ldots+N_{\mathrm{t}}+j} & : T_{\mathrm{m}_{j}}=g_{\mathrm{mo}}\left(T_{j}^{x y}\right)
\end{array}
$$

where indices $k, l, m, n, 1, i, j$ refer to gyro, position measurement units, vertical reference units, anemometer(s), current sensor, thrusters and tension measurements, respectively. The associated measurements are denoted $h_{k}, \mathbf{p}_{\mathrm{m}_{l}}, \mathbf{h}_{\mathrm{m}_{m}}$, $\mathbf{w}_{\mathrm{m}_{n}}, \mathbf{c}_{m}, u_{\mathrm{m}_{i}}, T_{\mathrm{m}_{j}}$.

Transformation of from actual sensor positions to ship's reference position is based on a rotation matrix, $\mathbf{R} \in \mathbb{R}^{2 \times 3}$, and a local position of the device, $\mathbf{l}_{l} \in \mathbb{R}^{3}$, as shown in Eq. (9). 
Table 3: Requirements of system arrangement for different DP classifications DNV (2014).

\begin{tabular}{llccc}
\hline & & \multicolumn{3}{c}{ Minimum requirements } \\
\cline { 3 - 5 } No. & Component & AUTS & AUT & AUTR \\
\hline$N_{\mathrm{t}}$ & Thrusters & No redundancy & No redundancy & Redundant \\
$N_{\mathrm{p}}$ & Position sensors & 1 & 2 & 3 \\
$N_{\mathrm{w}}$ & Anemometers & 1 & 1 & 2 \\
$N_{\mathrm{g}}$ & Heading sensors & 1 & 1 & 3 \\
$N_{\mathrm{r}}$ & Motion ref. units & 1 & 1 & 3 \\
\hline
\end{tabular}

The actual numbers of thrusters (actuators), sensors and measurement units depend on the class of the DP system. Table 3 shows the requirements for different classes according to the DNV-GL classification DNV (2014). Equivalent class notations exist from other classification societies, see IMO (1994) and later.

\section{Fault diagnosis}

The essence of analytic fault diagnosis is to establish relations to test whether measured and other known variables satisfy all relations that describe the system's normal behaviour. If this is not the case, some violation of normal behaviour has occurred, i.e. one or more faults are present in the system. Relations that can be used for such testing are referred to as redundancy relations.

Let a system be described by a set $\mathcal{X}$ of unknown variables, a set $\mathcal{K}$ of known variables and a set of constraints $\mathcal{C}$ on these variables. Then there may exist a set $\mathcal{C}_{m} \subseteq \mathcal{C}$ from which all variables in $\mathcal{X}$ can be determined. A system that has this property is said to have a complete matching on the unknown variables. If any constraints exist that were not used to obtain such matching, the set of unmatched constraints $\mathcal{C}_{u m} \subset \mathcal{C}$ may be used to test the consistency between known variables and the system's normal behaviour. Hence, redundancy relations are obtained with basis in the unmatched constraints.

Solving for unknown variables in a nonlinear system can be rather complex if done directly on the analytical form of the constraints. Structural analysis offers a significant shortcut. It is a method to determine possible ways to solve a set constraints without actually doing so. Making a graph representation of the relations between constraints and unknown variables makes it possible to seek through a graph to determine how one could solve for unknown variables. The result of a structural analysis is a receipt that, in symbolic form, describes how unknown variables could be calculated from known variables, using the system constraints. Analytical expressions are not used until after a complete structural solution is found. This dramatically reduces the complexity of finding redundancy relations for fault diagnosis.

The salient feature of the structural analysis approach is that graph theory exists that can be employed to find all possible ways the set of system constraints 
can be matched to unknown variables. A theoretic procedure was shown in the seminal paper Dulmage and Mendelsohn (1959). Later research has resulted in several algorithms to determine parity relations from structure graph information, summarised in Blanke et al. (2015) with significant efficiency improvements in algorithms reported in Krysander (2006), who finds all Minimally Structurally Overdetermined (MSO) subgraphs in a structure graph. Structural isolability properties may differ from residuals found from one complete matching to another, and an overall approach to selection of the sets of residuals was recently suggested in Svärd et al. (2013). This paper employs existing algorithms to find all complete matchings to revealed that violation in certain constraints in AUTS and AUT class systems will not be isolable, i.e. there are faults that can not be isolated. It will then be demonstrated how fault isolation can be achieved anyway by an active diagnosis approach. When such fault is detected, small magnitude reference changes to thrusters are used to show which constraints have become violated. Once the root cause of a fault is isolated, it is shown how fault-tolerant control is employed to handle the event.

\subsection{Structural domain analysis}

In the structural analysis approach, the variables in Eqs. (1) to (14) are classified as unknown, known input and known measured variables, respectively

$$
\begin{aligned}
X & =\left\{F_{i}, T_{j}^{x y}, \dot{\boldsymbol{\nu}}, \boldsymbol{\nu}, \dot{\boldsymbol{\eta}}, \boldsymbol{\eta}, \phi, \theta, \mathbf{p}, \psi, \mathbf{v}_{\mathrm{c}}, \mathbf{v}_{\mathbf{w}}\right\}, \\
K_{\mathrm{i}} & =\left\{u_{i}\right\} \\
K_{\mathrm{m}} & =\left\{h_{k}, \mathbf{p}_{\mathrm{m}_{l}}, T_{\mathrm{m}_{j}}, \mathbf{h}_{\mathrm{m}_{m}}, \mathbf{w}_{\mathrm{m}_{n}}, \mathbf{c}_{\mathrm{m}}, u_{\mathrm{m}_{i}}\right\} .
\end{aligned}
$$

The technique analyses the principal relations between these types of variables. The relations between variables are expressed through a generic model that explains the topology or structure in the set of constraints that define a behavioral model of our plant. The model, referred to as the structural model, of the system $(\mathcal{C}, \mathcal{Z})$ defines as a bi-partite graph $(\mathcal{C}, \mathcal{Z}, \mathcal{E})$, where $\mathcal{E} \subset \mathcal{C} \times \mathcal{Z}$ is a set of edges defined by $\left(c_{i}, z_{i}\right) \in \mathcal{E}$ if the variable $z_{i}$ appears in the constraint $c_{i}$. The structure graph of an AUT class positioning controlled vessel is depicted in Fig. 1. In this structure graph, a constraint is represented by a bar, an edge by a line and a variable by a circle.

One further defines a complete matching $\mathcal{M} \subset \mathcal{E}$ with respect to unknown variables as a subset of edges that orientates known variables towards constraints in order to solve for all unknown variables. It is noted that arrows on edges imply direction of causality, as illustrated in the structure graph (Fig. 1). The implication is that a variable must be solved for by following the direction of the arrow.

\subsection{What structural analysis offers}

Structural analysis is in essence a graph theoretical way that advises in which order a set of equations can be solved, i.e which unknown variable can be determined from which constraint. When a constraint is constructed such that one 


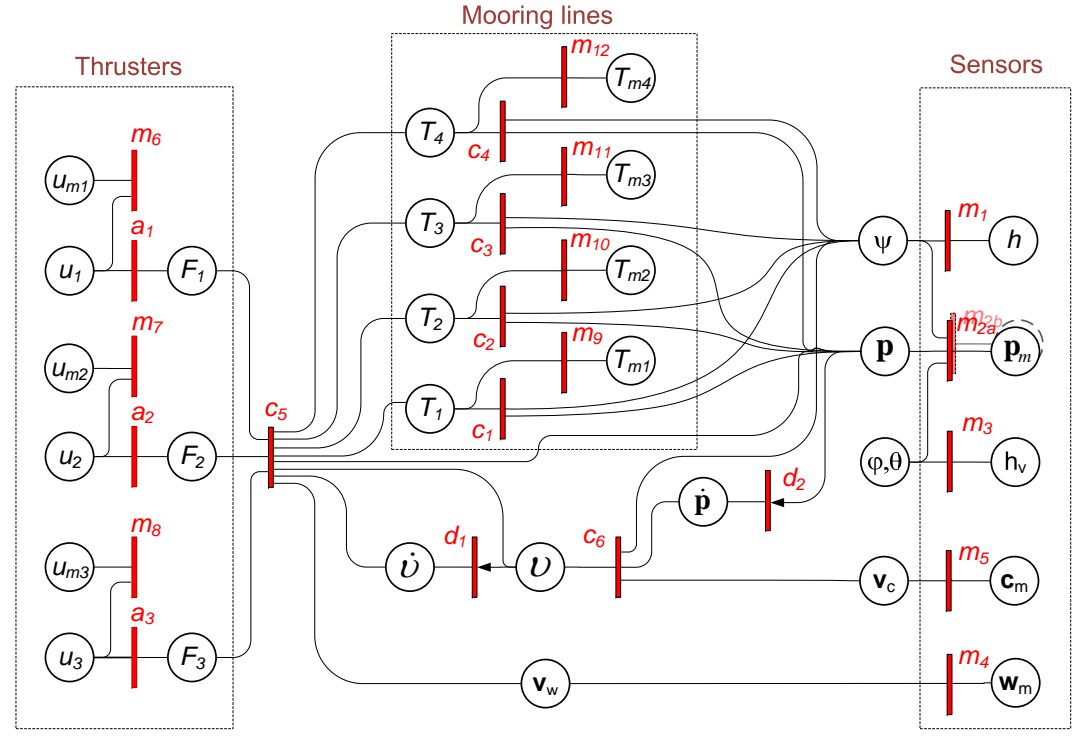

Figure 1: Generic structure graph for vessel configured to class AUT. The case shown has $N_{t}=3, N_{m}=4, N_{p}=2, N_{g}=1, N_{v}=1, N_{w}=1$. An AUTS vessel would have $N_{p}=1$. 
or more of the variables that are included in the constraint cannot be calculated from the constraint, but others can, we define a direction of calculation in a graph. As example in $c: x_{1}=g\left(x_{2}\right), x_{2}$ can only be calculated from $c$ if $g$ is invertible. If calculation is one-way only, we denote $c: x_{1} \leftarrow g\left(x_{2}\right)$ when performing the structural analysis.

\subsubsection{Violation of constraints}

In structural analysis, a fault is defined as a violation of a constraint. Such violation will affect a parity relation if this parity relation is constructed from that constraint. If a fault affects the residual vector, it is said to be structurally detectable. If a fault affects the unique pattern of the residual vector's elements, it is structurally isolable.

It is noted that the results from structural analysis are necessary but not sufficient conditions for analytical property. E.g. the structural isolability does not imply the isolability of a real fault while the isolability of a real fault does imply the structural isolability.

\subsubsection{Dealing with vector relations}

Structural analysis was originally developed to deal with sets of scalar variables and functions. In a marine setting, some variable are conveniently treated as vectors, e.g position $\mathbf{p}$ has components in North and East of the Earth-fixed navigation frame, $\mathbf{p}=\left[p_{N}, p_{E}\right]^{T}$ and velocity is $\mathbf{v}=\left[v_{N}, v_{E}\right]^{T}$. Relating velocity to position $\dot{\mathbf{p}}=\mathbf{v}$ therefore constitutes two uncoupled linear equations to which the structural analysis tools immediately apply.

Transformation in the horizontal plane from body to navigation frame $c$ : $\mathbf{v}^{n}=\mathbf{R}_{n b}(\psi) \mathbf{v}^{b}$ includes the $2 \times 2$ rotation matrix $\mathbf{R}_{n b}(\psi)$, both of the velocity vectors are calculable from $c$, since the rotation is invertible. Some automated tools, including SaTool Blanke and Lorentzen (2006), cannot automate the calculation of the angle of rotation from such constraint. This is a software technicality that is dealt with by declaring, to the software, that $\psi$ cannot be determined from $c$.

In some cases, vectors need be written in component form. In a scalar product between vectors $\mathbf{a}$ and $\mathbf{b} c: 0=\mathbf{a b}^{T}$, one vector cannot be uniquely determined even if the other is known, since any vector, perpendicular to the known one, will satisfy the constraint. However, writing the equation in component form, $0=a_{1} b_{1}+a_{2} b_{2}+a_{3} b_{3}$, will allow the structural analysis tools to solve for one element if the others are known.

Convenience and concerns for brevity have dictated that vector notation is used when possible in this paper.

\subsection{Redundancy relations for class AUT vessel}

From the discussion above, the entire set of constraints are, for a class AUT vessel with 4 mooring lines $\left(N_{m}=4\right), 3$ thrusters $\left(N_{t}=3\right)$ and class AUT instrumentation: 
Table 4: Constraints for AUT class vessel with $N_{t}=3$ thrusters,

$N_{m}=4$ mooring lines and 2 position measurements

$$
\begin{array}{l|l}
a_{i} & F_{i}=\mathbf{g}_{p}\left(u_{i}\right) \quad \text { for } i=1 \ldots N_{t} \\
c_{j} & T_{j}=\mathbf{g}_{m_{j}}(\mathbf{p}, \psi) \quad \text { for } j=1 \ldots N_{m} \\
c_{5} & \mathbf{M} \dot{\boldsymbol{\nu}}+\mathbf{C} \boldsymbol{\nu}=\mathbf{g}_{w}\left(v_{w}\right)+\sum_{i=1}^{N_{t}}\left(\mathbf{A}_{b t}^{i} g_{p}\left(u_{i}\right)\right)+\sum_{j=1}^{N_{m}}\left(\mathbf{A}_{b m}^{j} T_{m_{j}}\right) \\
c_{6} & \dot{\mathbf{p}}=\mathbf{v}_{c}+\mathbf{R}(\psi) \boldsymbol{\nu} \\
d_{1} & \dot{\boldsymbol{\nu}}=\frac{d}{d t} \boldsymbol{\nu} \\
d_{2} & \dot{\mathbf{p}}=\frac{d}{d t} \mathbf{p} \\
m_{1} & \psi_{m}=\psi \\
m_{2 a} & \mathbf{p}_{m 1}=\mathbf{p}+R((\phi, \theta), \psi) \mathbf{l} \\
m_{2 b} & \mathbf{p}_{m 2}=\mathbf{p}+R((\phi, \theta), \psi) \mathbf{l} \\
m_{3} & \left(\phi_{m}, \theta_{m}\right)=(\phi, \theta) \\
m_{4} & \mathbf{w}_{m}=\mathbf{v}_{w} \\
m_{5} & \mathbf{c}_{m}=\mathbf{v}_{c} \\
m_{5+i} & u_{m i}=u_{i} \text { for } i=1 \ldots N_{t} \\
m_{8+j} & T_{m j}=T_{j} \text { for } j=1 \ldots N_{m}
\end{array}
$$

The structural analysis given the constraints in Table 4 with unknown variables listed in Eq.15 and known ones in Eqs. 16 - 17. Analysis is done using the SaTool software Blanke and Lorentzen (2006) where different algorithms are available to find matchings and MSO sets. A set of analytical redundancy relations (AAR) is generated as the result of the structural analysis. Each complete matching or MSO set will define a set of relations, see Table 5, that shows which constraint is used to calculate each of the unknown variables. A 0 in the table indicates an unmatched constraint that can be used as an ARR.

Table 5: A complete matching of the AUT class system in Table 4

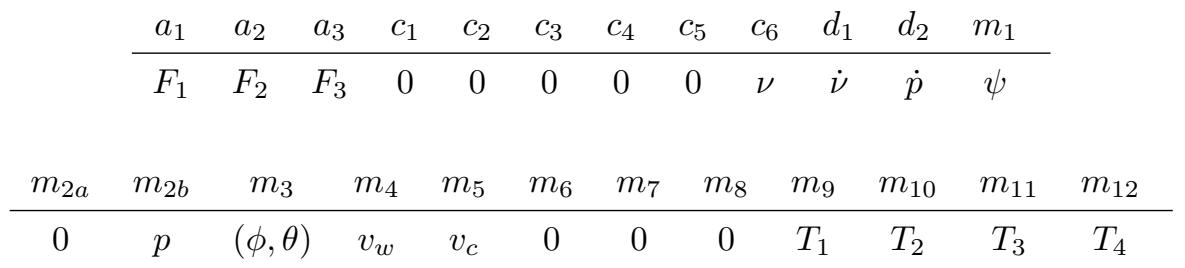

The complete matching of Table 5 gives a set of 9 ARRs. Each ARR provides a balance that must be present between left and right hand sides of the ARR. Forming the difference between the two sides of an ARR gives a residual, which is zero, or close to, when no constraint in the ARR is violated; it is non-zero if a constraint is violated.

For the AUT class system, the complete matching shown in Table 5, gives ARRs corresponding to the unmatched constraints $\left\{c_{1}, c_{2}, c_{3}, c_{4}, c_{5}, m_{6}, m_{7}, m_{8}\right\}$. Automated back-tracking Blanke et al. (2015), Laursen et al. (2008), to known variables give an automated procedure to generate the ARRs in symbolic form Blanke and Lorentzen (2006). Using $h_{v}=(\phi, \theta)$ and $h=\psi$ for convenience, the 


$$
\begin{aligned}
& \operatorname{arr}_{1}: \quad 0=c_{1}\left(m_{9}\left(T_{m 1}\right), m_{2 b}\left(p_{m 2}, m_{3}(h v), m_{1}(h)\right), m_{1}(h)\right) \\
& \operatorname{arr}_{5}: \quad 0=c_{5}\left(a_{1}\left(u_{1}\right), a_{2}\left(u_{2}\right), a_{3}\left(u_{3}\right), m_{9}\left(T_{m 1}\right), m_{10}\left(T_{m 2}\right), m_{11}\left(T_{m 3}\right),\right. \\
& m_{12}\left(T_{m 4}\right), d_{1}\left(c_{6}\left(d_{2}\left(m_{2 b}\left(p_{m 2}, m_{3}(h v), m_{1}(h)\right)\right), m_{1}(h), m_{5}\left(c_{m}\right)\right)\right), \\
& \left.c_{6}\left(d_{2}\left(m_{2 b}\left(p_{m 2}, m_{3}(h v), m_{1}(h)\right)\right), m_{1}(h), m_{5}\left(c_{m}\right)\right), m_{4}\left(w_{m}\right)\right) \\
& \operatorname{arr}_{6}: \quad 0=m_{6}\left(u_{1}, u_{m 1}\right) \\
& \operatorname{arr}_{9}: \quad 0=m_{2 a}\left(p_{m 1}, m_{3}(h v), m_{2 b}\left(p_{m 2}, m_{3}\left(h_{v}\right), m_{1}(h)\right), m_{1}(h)\right)
\end{aligned}
$$

Replacing the left hand side zero in $\operatorname{arr}_{i}: 0=c_{j}(\ldots)$ by a residual $r_{i}$ provides a basis for diagnosis. A residual remains zero if no violation of any constraint in the associated ARR is present. Structural detectability (SD) and - isolability (SI) follows from the pattern in which constraints participate in the calculation of residuals. SD of $c_{i}$ follows if $c_{i}$ participates in the calculation of any of the residuals, $r_{j} \in \boldsymbol{r}$. SI follows when $c_{i}$ has a unique signature in $\boldsymbol{r}$, see e.g. Blanke and Staroswiecki (2006), Blanke et al. (2015) for concise definitions.

\subsubsection{Structural detectability and isolability}

The number of AAR's available from one complete matching is less or equal to the number of constraints less the number of unknown variables, hence different sets of residuals will be available for different DP class vessels, and detectability and isolability properties differ as well. As example, Table 6 shows AUTS and AUT vessels with $N_{\mathrm{m}}=4$ mooring lines and $N_{\mathrm{t}}=3$ thrusters. The AUTS vessel has eight ARR relations from one complete matching. AAR's created as MSO sets Krysander (2006) is an alternative where 96 AAR's are generated. In general, detectability will be the same for the two ways of finding ARRs, but isolability can be enhanced by using the MSO solution, at the expense of running more ARRs in parallel than with the single matching set. In the DP case, the use of MSO sets does not improve isolability for this particular system.

Table 6 shows the structural detectability and isolability if a constraint should be violated. Constraints are shown in the first row; the corresponding physical components in the second; structural detectability $d$ and isolability $i$ are shown in the bottom rows.

\subsubsection{Results for multiple faults}

Analysis of cases of multiple faults is important when a DP vessel is desired to continue operation despite the presence of one or more faults in the system. Detectability and isolability are essential for the FTC solution in each case since a controller used in case of fault(s) would be unsafe if it relies on sensors for which a failure could not be detected Blanke (2005). 
Table 6: Analysis of single fault cases for vessel of DP class AUTS and AUT.

\begin{tabular}{|c|c|c|c|c|c|c|c|c|c|c|c|}
\hline Constraint & $\mathrm{a}_{i}$ & $\mathrm{c}_{i}$ & $c_{5}$ & $\mathrm{~m}_{1}$ & $\mathrm{~m}_{2 a}$ & $\mathrm{~m}_{2 b}$ & $\mathrm{~m}_{3}$ & $\mathrm{~m}_{4}$ & $\mathrm{~m}_{5}$ & $\mathrm{~m}_{5+i}$ & $\mathrm{~m}_{5+N_{\mathrm{t}}+j}$ \\
\hline $\begin{array}{l}\overrightarrow{\overrightarrow{0}} \\
\stackrel{0}{0} \\
\text { : } \\
\text { : } \\
0\end{array}$ & 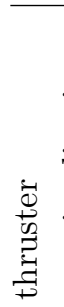 & 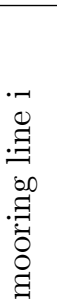 & 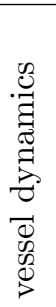 & $\underset{\overbrace{0}}{\stackrel{0}{0}}$ & 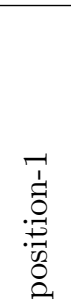 & 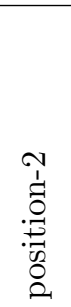 & 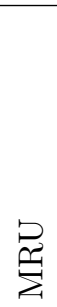 & 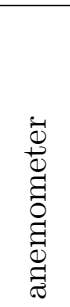 & 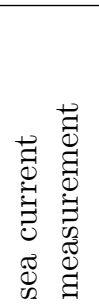 & 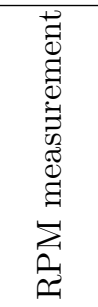 & 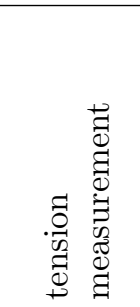 \\
\hline AUTS & $\mathrm{d}$ & $\mathrm{i}$ & $\mathrm{d}$ & $\mathrm{d}$ & $\mathrm{d}$ & - & $\mathrm{d}$ & $\mathrm{d}$ & $\mathrm{d}$ & $\mathrm{i}$ & $\mathrm{i}$ \\
\hline AUT & d & $\mathrm{i}$ & $\mathrm{d}$ & d & $\mathrm{i}$ & $\mathrm{i}$ & d & d & d & $\mathrm{i}$ & $\mathrm{i}$ \\
\hline
\end{tabular}

Table 7: Analysis of two simultaneous faults for a DP vessel of AUTS class.

\begin{tabular}{lcccccccccccc}
\hline$f_{1}$ & $f_{2}$ & $\mathrm{a}_{i}$ & $\mathrm{c}_{1}$ & $\mathrm{c}_{2}$ & $\mathrm{c}_{5}$ & $\mathrm{~m}_{1}$ & $\mathrm{~m}_{2}$ & $\mathrm{~m}_{3}$ & $\mathrm{~m}_{4}$ & $\mathrm{~m}_{5}$ & $\mathrm{~m}_{5+i}$ & $\mathrm{~m}_{5+N_{\mathrm{t}}+j}$ \\
\hline $\mathrm{a}_{i}$ & - & $\mathrm{d}$ & $\mathrm{d}$ & 0 & $\mathrm{~d}$ & $\mathrm{~d}$ & $\mathrm{~d}$ & 0 & 0 & $\mathrm{i}$ & $\mathrm{d}$ \\
$\mathrm{c}_{1}$ & $\mathrm{~d}$ & - & $\mathrm{i}$ & $\mathrm{d}$ & $\mathrm{d}$ & $\mathrm{d}$ & $\mathrm{d}$ & $\mathrm{d}$ & $\mathrm{d}$ & $\mathrm{i}$ & $\mathrm{d}$ \\
$\mathrm{c}_{5}$ & 0 & $\mathrm{~d}$ & $\mathrm{~d}$ & - & $\mathrm{d}$ & $\mathrm{d}$ & $\mathrm{d}$ & 0 & 0 & $\mathrm{i}$ & $\mathrm{d}$ \\
$\mathrm{m}_{2}$ & $\mathrm{~d}$ & $\mathrm{i}$ & $\mathrm{i}$ & $\mathrm{d}$ & 0 & - & 0 & $\mathrm{~d}$ & $\mathrm{~d}$ & $\mathrm{i}$ & $\mathrm{i}$ \\
$\mathrm{m}_{3}$ & $\mathrm{~d}$ & $\mathrm{i}$ & $\mathrm{i}$ & $\mathrm{d}$ & 0 & 0 & - & $\mathrm{d}$ & $\mathrm{d}$ & $\mathrm{i}$ & $\mathrm{i}$ \\
$\mathrm{m}_{4}$ & 0 & $\mathrm{~d}$ & $\mathrm{~d}$ & 0 & $\mathrm{~d}$ & $\mathrm{~d}$ & $\mathrm{~d}$ & - & 0 & $\mathrm{i}$ & $\mathrm{d}$ \\
$\mathrm{m}_{5+i}$ & $\mathrm{~d}$ & $\mathrm{i}$ & $\mathrm{i}$ & $\mathrm{d}$ & $\mathrm{d}$ & $\mathrm{d}$ & $\mathrm{d}$ & $\mathrm{d}$ & $\mathrm{d}$ & - & $\mathrm{i}$ \\
$\mathrm{m}_{5+N_{\mathrm{t}}+1}$ & $\mathrm{~d}$ & $\mathrm{~d}$ & $\mathrm{i}$ & $\mathrm{d}$ & $\mathrm{d}$ & $\mathrm{d}$ & $\mathrm{d}$ & $\mathrm{d}$ & $\mathrm{d}$ & $\mathrm{i}$ & - \\
\hline
\end{tabular}

Table 7 shows the structural results for the AUTS class configuration with a particular fault $f_{1}$ already present and a second fault $f_{2}$ occurs. As seen from the table, if a fault already occurred in a mooring line $\left(c_{1}\right)$, a further fault in the tension measurement unit $\left(m_{9}\right)$ of this line is only detectable instead of isolable. If a fault already occurred in a tension measurement unit, a fault in the corresponding mooring line would still be structurally isolable. Table 8 shows a few cases of three simultaneous faults.

The table illustrates which structural faults are detectable and isolable after two faults have occurred. In the second row block, thruster $a_{i}$ first failed, then thruster RPM measurement $m_{5+i}$ fails (first line) or tension measurement $j m_{5+N_{t}+j}$ fails (second line). The consequence of the RPM fault is that further faults in position from current and body velocity $c_{6}$, anemometer $m_{4}$ or sea current $m_{5}$ could not be detected. The consequence of the second fault being in tension sensor for line 2 is that, furthermore, a line defect in line 2 
would be invisible to the fault detection system, when using passive fault detection techniques. Active fault isolation is described later in this paper. These information are used in the FTC supervisor algorithms that determine which remedial action should be initiated in each case of single or multiple defects.

Table 8: Analysis of three simultaneous faults for a DP vessel of AUTS class.

\begin{tabular}{llllllllllllll}
\hline$f_{1}$ & & $f_{3}$ & $\mathrm{a}_{i}$ & $\mathrm{c}_{2}$ & $\mathrm{c}_{j \neq 2}$ & $\mathrm{c}_{6}$ & $\mathrm{~m}_{1}$ & $\mathrm{~m}_{2}$ & $\mathrm{~m}_{3}$ & $\mathrm{~m}_{4}$ & $\mathrm{~m}_{5}$ & $\mathrm{~m}_{5+i}$ & $\mathrm{~m}_{5+N_{\mathrm{t}+j}}$ \\
\hline \multirow{3}{*}{$\mathrm{c}_{2}$} & $\mathrm{a}_{i}$ & - & - & $\mathrm{d}$ & 0 & $\mathrm{~d}$ & $\mathrm{~d}$ & $\mathrm{~d}$ & 0 & 0 & $\mathrm{i}$ & 0 \\
& $\mathrm{~m}_{2}$ & $\mathrm{~d}$ & - & $\mathrm{i}$ & $\mathrm{d}$ & 0 & - & 0 & $\mathrm{~d}$ & $\mathrm{~d}$ & $\mathrm{i}$ & $\mathrm{d}$ \\
& $\mathrm{m}_{5+N_{\mathrm{t}}+j}$ & 0 & - & $\mathrm{d}$ & 0 & $\mathrm{~d}$ & $\mathrm{~d}$ & $\mathrm{~d}$ & 0 & 0 & $\mathrm{i}$ & - \\
\hline \multirow{2}{*}{$\mathrm{a}_{i}$} & $\mathrm{~m}_{5+i}$ & - & $\mathrm{d}$ & $\mathrm{d}$ & 0 & $\mathrm{~d}$ & $\mathrm{~d}$ & $\mathrm{~d}$ & 0 & 0 & - & $\mathrm{d}$ \\
& $\mathrm{m}_{5+N_{\mathrm{t}}+j}$ & - & 0 & $\mathrm{~d}$ & 0 & $\mathrm{~d}$ & $\mathrm{~d}$ & $\mathrm{~d}$ & 0 & 0 & $\mathrm{i}$ & - \\
\hline
\end{tabular}

This analysis has considered violation of one or more constraints. Some physical faults may affect more than one constraint. In such cases, the multiple violations are defined in the SaTool software and re-analysis is done.

\subsection{Analytical domain analysis}

This design step includes: obtain residuals in analytical form; ensure residuals are causal; model faults as signals; and investigate diagnosability properties of physical faults.

\subsubsection{Residuals in analytical form}

The symbolic form (Eq. 18) advise the way ARRs are to be calculated. Inserting the analytical form of constraints hence makes it possible to autogenerate residuals. For the AUT class, $N_{r}=9$ residuals are auto-generated in this way: $r_{1} \ldots r_{4}$ express a force balance of each of the mooring lines; $\mathbf{r}_{5}$ the force balance on the vessel; $r_{6} \ldots r_{8}$ the difference between command and measured rotational speed for each of the thrusters; $r_{9}$ the deviation between the two position sensors. 
The residuals read, in analytical form in the continuous time domain,

$$
\begin{aligned}
r_{j}(t) & =T_{m i}(t)-\left(g_{m_{j}}\left(\mathbf{p}_{m 1}(t)-\mathbf{R}_{n b}(t) \mathbf{l}, \psi_{m}(t)\right)\right), j=1 \ldots N_{m} \\
\mathbf{r}_{5}(t) & =\mathbf{M} \mathbf{R}_{b n}(t) \frac{d}{d t}\left(\frac{d}{d t}\left(\mathbf{p}_{m 1}(t)-\mathbf{R}_{n b}(t) \mathbf{l}\right)-\mathbf{c}_{m}(t)\right) \\
& -\mathbf{M} \boldsymbol{\omega}(t) \times\left(\frac{d}{d t}\left(\mathbf{p}_{m 1}(t)-\mathbf{R}_{n b}(t) \mathbf{l}\right)-\mathbf{c}_{m}(t)\right) \\
& +\mathbf{C} \mathbf{R}_{b n}(t)\left(\frac{d}{d t}\left(\mathbf{p}_{m 1}(t)-\mathbf{R}_{n b}(t) \mathbf{l}\right)-\mathbf{c}_{m}(t)\right) \\
& -\left(\mathbf{g}_{w}\left(\mathbf{w}_{m}(t)\right)+\sum_{i=1}^{N_{t}}\left(\mathbf{A}_{b t}^{i} g_{p}\left(u_{i}(t)\right)\right)+\sum_{j=1}^{N_{m}}\left(\mathbf{A}_{b m}^{j} T_{m_{j}}(t)\right)\right) \\
r_{5+i}(t) & =u_{m i}(t)-u_{i}(t) \text { for } i=1 \ldots N_{t} \\
r_{9}(t) & =\mathbf{p}_{m 1}(t)-\mathbf{p}_{m 2}(t)
\end{aligned}
$$

\subsubsection{Physical faults}

A physical fault $\mathbf{f}_{j}$ impacting one but possibly a subset of the constraints simultaneously,

$$
\mathbf{f}_{j} \Rightarrow\left\{c_{i} \neq 0\right\} i \in\left[1, \ldots, N_{\text {arr }}\right] .
$$

In our case, physical faults of interest are: fault in any sensor; fault in any thruster; fault in any mooring line, and according to Table 6, each such component fault origin in a single constraint. Some component faults can be modelled as additive signals, $\mathbf{p}_{m i}=\mathbf{p}+\mathbf{f}_{p i}$, for a position sensor fault, others as multiplicative, $g_{p}\left(u_{i}\right)=\left(1-\mathbf{f}_{p i}\right) g_{p}\left(u_{i}\right)$, for a thruster force generation defect.

Common mode fault originating from faults in power system or physical infrastructure (fire, partial flooding, partial loss of power, local area network disruption) are possible to model and analyse but this has not been within the scope of this study.

\subsubsection{Fault detectability and isolability}

Using the definitions in Blanke et al. (2015), a fault $f(t)$ occurs a $t=t_{0}$ and has bounded magnitude, $|f(t)| \leq \bar{f}$. This fault is strongly detectable in the residual vector if a stable residual generator exists with the property:

$$
\forall t \geqq t_{0}, 0<\left|f_{j}(t)\right| \leq \bar{f} \Longrightarrow|r(t)| \neq 0, \forall t \geq t_{0}
$$

A fault is weakly detectable if a stable residual generator exists with the property:

$$
\exists t_{0}<t_{1}<t_{2}: \forall t \geqq t_{0},\left|f_{i}(t)\right| \neq 0 \Rightarrow \exists t_{1}, t_{2}:|r(t)| \neq 0 \text { for } t_{1}<t<t_{2} .
$$

Table 9 shows the relation between defects in constraints, each representing a physical component, and residuals in a dependency table. The following symbols are used: detectability, $d$; isolability, $i$; strong, $s$; and weak, $w$. The table shows that all faults are strongly detectable, the majority are furthermore strongly isolable, but thruster faults are only detectable. 
Table 9: Residuals' dependency of individual violation of constraints for AUT class with $N_{t}=3, N_{m}=4, N_{p}=2, N_{g}=1, N_{v}=1, N_{w}=1$

\begin{tabular}{|c|c|c|c|c|c|c|c|c|c|c|c|c|c|c|c|c|c|c|c|c|}
\hline- & $a_{1}$ & $a_{2}$ & $a_{3}$ & $\mathrm{c}_{1}$ & $\mathrm{c}_{2}$ & $c_{3}$ & $\mathrm{c}_{4}$ & $\mathrm{~m}_{1}$ & $\mathrm{~m}_{2}^{a}$ & $\mathrm{~m}_{2}^{b}$ & $\mathrm{~m}_{3}$ & $\mathrm{~m}_{4}$ & $\mathrm{~m}_{5}$ & $\mathrm{~m}_{6}$ & $\mathrm{~m}_{7}$ & $\mathrm{~m}_{9}$ & $\mathrm{~m}_{9}$ & $\mathrm{~m}_{10}$ & $\mathrm{~m}_{11}$ & $\mathrm{~m}_{12}$ \\
\hline $\begin{array}{l}\text { Z्वि } \\
\text { ठ }\end{array}$ & $\underset{\exists}{\exists}$ & $\stackrel{N}{\exists}$ & $\begin{array}{l}\infty \\
\stackrel{A}{\varpi}\end{array}$ & $\stackrel{\Xi}{\Xi}$ & $\underset{\Xi}{\stackrel{N}{\Xi}}$ & $\begin{array}{l}\infty \\
\stackrel{\Xi}{\Xi}\end{array}$ & $\begin{array}{l}+ \\
\Xi \\
\Xi\end{array}$ & $\stackrel{\circ}{\vec{C}}$ & $\begin{array}{l}\overrightarrow{1} \\
\tilde{n} \\
0 \\
0\end{array}$ & $\begin{array}{l}N \\
n \\
0 \\
2\end{array}$ & $\stackrel{D}{\overbrace{}^{\prime}}$ & $\stackrel{\vec{Z}}{3}$ & $\exists$ & $\sum_{\sum_{1}^{\prime}}^{-}$ & $\sum_{\sum_{n}^{1}}^{N}$ & $\sum_{\infty}^{\infty}$ & 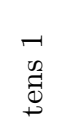 & $\begin{array}{l}N \\
\text { Dี } \\
\Phi\end{array}$ & 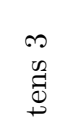 & 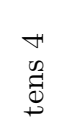 \\
\hline$r_{1}$ & & & & $\mathrm{~s}$ & & & & $\mathrm{~s}$ & $\mathrm{~s}$ & & $\mathrm{~s}$ & & & & & & $\mathrm{~s}$ & & & \\
\hline$r_{2}$ & & & & & $\mathrm{~s}$ & & & $\mathrm{~s}$ & $\mathrm{~s}$ & & $\mathrm{~s}$ & & & & & & & $\mathrm{~s}$ & & \\
\hline$r_{3}$ & & & & & & $\mathrm{~s}$ & & $\mathrm{~s}$ & $\mathrm{~s}$ & & $\mathrm{~s}$ & & & & & & & & $\mathrm{~s}$ & \\
\hline$r_{4}$ & & & & & & & $\mathrm{~s}$ & $\mathrm{~S}$ & $\mathrm{~S}$ & & $\mathrm{~S}$ & & & & & & & & & $\mathrm{~s}$ \\
\hline $\mathbf{r}_{5}$ & $\mathrm{~s}$ & $\mathrm{~s}$ & $\mathrm{~S}$ & & & & & & $\mathrm{w}$ & $\mathrm{w}$ & $\mathrm{w}$ & $\mathrm{S}$ & $\mathrm{S}$ & & & & $\mathrm{s}$ & $\mathrm{s}$ & $\mathrm{S}$ & $\mathrm{s}$ \\
\hline$r_{6}$ & & & & & & & & & & & & & & $\mathrm{~s}$ & & & & & & \\
\hline$r_{7}$ & & & & & & & & & & & & & & & $\mathrm{~s}$ & & & & & \\
\hline$r_{8}$ & & & & & & & & & & & & & & & & $\mathrm{~s}$ & & & & \\
\hline$r_{9}$ & & & & & & & & & $\mathrm{~S}$ & $\mathrm{~S}$ & & & & & & & & & & \\
\hline $\mathrm{d} / \mathrm{i}$ & $\mathrm{d}$ & $\mathrm{d}$ & $\mathrm{d}$ & $\mathrm{i}$ & $\mathrm{i}$ & $\mathrm{i}$ & $\mathrm{i}$ & $\mathrm{d}$ & i & $\mathrm{i}$ & $\mathrm{d}$ & $\mathrm{d}$ & $\mathrm{d}$ & $\mathrm{i}$ & $\mathrm{i}$ & $\mathrm{i}$ & $\mathrm{i}$ & $\mathrm{i}$ & $\mathrm{i}$ & $\mathrm{i}$ \\
\hline $\mathrm{w} / \mathrm{s}$ & $\mathrm{s}$ & $\mathrm{s}$ & $\mathrm{S}$ & $\mathrm{s}$ & $\mathrm{S}$ & $\mathrm{s}$ & $\mathrm{s}$ & $\mathrm{S}$ & $\mathrm{S}$ & $\mathrm{S}$ & $\mathrm{S}$ & $\mathrm{S}$ & $\mathrm{S}$ & $\mathrm{S}$ & $\mathrm{S}$ & $\mathrm{s}$ & $\mathrm{S}$ & $\mathrm{S}$ & $\mathrm{S}$ & $\mathrm{s}$ \\
\hline
\end{tabular}

\subsubsection{Causality of residuals}

ARRs in general contain derivatives, in this case Eq. 20, and filtering may need be employed to reach a causal implementation of a residual generator; either through linear lowpass filtering of appropriate order or by using a modelbased feedback structure, an observer or a Kalman filter.

Applying a simple linear filter $H(s)$ Eq. 20 gives the modified (filtered) residual, $\mathbf{r}_{5}^{f}(t)$, with the Laplace domain equivalent,

$$
\mathbf{r}_{5}^{\mathrm{f}}(s)=H(s) \mathbf{r}_{5}(s) .
$$

The reduction in variance due to filtering is easily calculated using standard methods, either by finding the resulting variance through integration,

$$
\sigma_{r_{5}, f}^{2}=\int_{-\infty}^{\infty} H(j \omega) H(-j \omega) S_{\mathbf{r}_{5} \mathbf{r}_{5}}(j \omega) d \omega
$$

where $S_{\mathbf{r}_{5} \mathbf{r}_{5}}$ denotes the spectrum of the unfiltered residual, or by solving the Lyapunov equation if the filter and any non-whiteness in the residual are represented as a state-space form, Blanke et al. (2015). The resulting variance and correlating structure are important for the change detection properties.

\subsection{Discussion}

The approach to design residual generators, as outlined above, is comparable with other methods for generating residuals for fault diagnosis. FDI observers for linear systems, see Garcia and Frank (1997) and references herein, or for nonlinear systems Persis and Isidori (2001), Besançon (2003) and later extensions, obtain dynamic filters as generators of residuals. These observers mask 
a disturbance from the residual. The residuals obtained from structural analysis have the same property since, by including a disturbance as an unknown variable, the obtained matching will use one of the constraints to match this unknown variable, and the AARs will be independent of the unknown input.

\subsection{Change detection}

Once the residuals are obtained, a change detection algorithm decides whether a change can be confirmed, the $\mathcal{H}_{1}$ hypothesis, or the normal case can be confirmed, the $\mathcal{H}_{0}$ hypothesis. If a fault causes a known change in the residuals a classical cumulative sum (CUSUM) test, Basseville and Nikiforov (1993), is a simple and efficient means for hypothesis testing. For the further analysis, let the discrete-time equivalents to the residuals Eqs. $19-22$ be the sampled versions of $r_{i}(t)$. With sampling time $T_{s}$, these are denoted $r\left(k T_{s}\right)$ or $r(k)$, for brevity.

With strong detectability of faults in residuals, change from normal $\mathcal{H}_{0}$ to not-normal $\mathcal{H}_{1}$, is seen as a change in mean from $\boldsymbol{\mu}_{0}$ to $\boldsymbol{\mu}_{1}$ for the $i^{\text {th }}$ residual,

$$
\begin{aligned}
& \mathcal{H}_{0}: \mathbf{r}(k)=\boldsymbol{\mu}_{0}+\mathbf{w}(k) \\
& \mathcal{H}_{1}: \\
& \mathbf{r}(k)=\boldsymbol{\mu}_{1}+\mathbf{w}(k) .
\end{aligned}
$$

A recursive form of the CUSUM test for each of the $i$ components of the residual vector, the scalar test case, gives the test statistics,

$$
g_{i}(k)=\frac{\Delta \mu_{i}}{\sigma_{i}^{2}} \max \left(0, g_{i}(k-1)+r_{i}(k)-\mu_{0 i}-\frac{\Delta \mu_{i}}{2}\right),
$$

where $\mu_{0 i}$ and $\sigma_{i}^{2}$ are the mean and variance, respectively, and $\Delta \mu_{i}=\mu_{1 i}-\mu_{0 i}$ is the change of the mean of the Gaussian sequence to be detected. When the decision function $g_{i}(k)$ exceeds a threshold $h, \mathcal{H}_{1}$ is assumed and an alarm is triggered.

A very useful measure for design of a CUSUM test is the average run length (ARL), see Basseville and Nikiforov (1993). The ARL tells two essential things. First, under $\mathcal{H}_{0}$ (no fault), how long is the average time until the test statistics $g(k)$ exceeds a threshold $h$. This is the mean time between false alarms. Second, under $\mathcal{H}_{1}$ (fault is present), which is the average time until $g(k)$ exceeds $h$. This is the average time to detect. The important parameters in the ARL test are the change magnitude of the test statistics divided by its' standard deviation $\frac{\mu_{s}}{\sigma_{s}}$, and the threshold $h$ for the test. Filtering of the residual will impact the variance of the test statistics. Therefore filtering is used as a means to make CUSUM tests more sensitive. Fig. 2 shows the calculated time to detect and time between false alarms for the residual $r_{5}^{\mathrm{f}}$, using the test statistic Eq. 27. To design the detector, the filter cut-off frequencies were set to $\omega_{c}=1.6,2.0$ and $2.4 \mathrm{rad} / \mathrm{s}$ in the theoretical calculation. The variance on residuals was measured under nominal conditions of wave load and sensor noise by running a simulation of a vessel, and subsequently by running model tests under the same conditions. The simulation and experiment setup are described in Section 6 . 

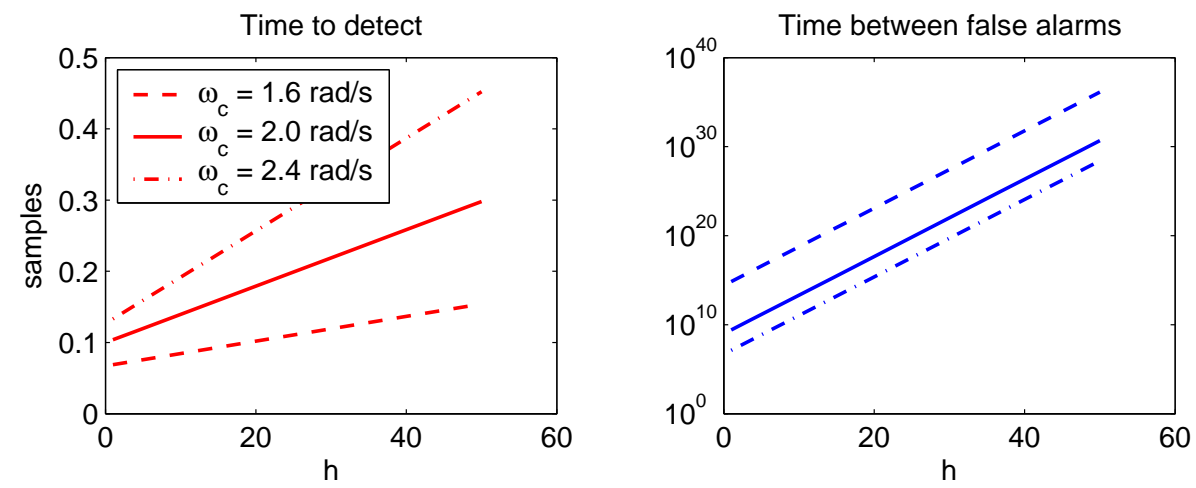

Figure 2: Time to detect and time between false alarm according the the ARL for $r_{5}^{\mathrm{f}}$ as function of threshold $h$ if a second order low-pass filter $H(s)$ is used with two co-located eigenvalues at $s=j \omega_{c}$. Time to detect will be one sample in all cases within the range of the nondimentional threshold, $h$ plotted along the abscissa axis.
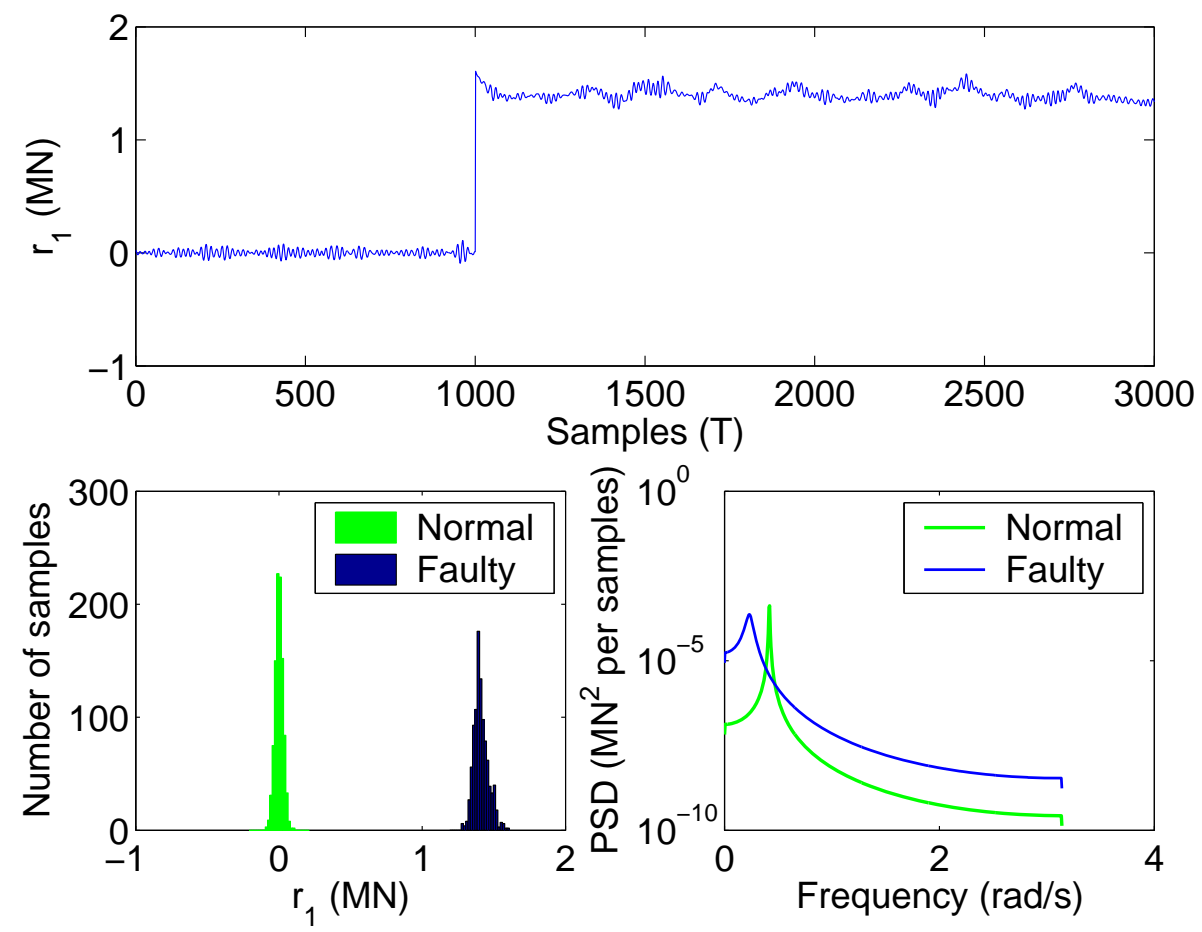

Figure 3: Residual $r_{1}$ and its histogram and power spectrum. 
Fig. 3 shows the time history of $r_{1}$ and its histogram and power spectrum when a line break occurs in mooring line 1 . It was observed that the distributions of $r_{1}$ in faultless and in faulty conditions can be assumed to be Gaussian with different means and the same variance.

\subsection{Active isolation}

Once a fault is isolated, the system has to handle the fault with appropriate control actions. Since thruster faults could only be detected, and such fault could only be isolated to the group of thrusters, other means are needed to isolate a faulty thruster and accommodate for the fault in the control system.

The concept of active fault isolation can obtain this. To exemplify the concept, consider thruster faults, that can only be groupwise isolated. If a fault is isolated to be within either of the thrusters, but a specific thruster cannot be identified as faulty, small dedicated test signals are added to thruster setpoint. When possible, such perturbations are chosen such that the resulting thrust would be in the nullspace of the thruster configuration matrix $\mathbf{A}_{b t}$. With this choice of perturbation, the resulting motion would be zero if all thrusters were fault-free. A small vessel motion will be the result when one of the thrusters has a defect, i.e. thrust produced differs from thrust demanded. This vessel motion will be correlated with the perturbation signals. Simultaneously, elements in the residual vector will have a variation that is correlated with the perturbation signals. In order that active fault isolation can be achieved, the behaviour of input to output and input to residuals propagation of signals need to have certain properties, that can be described through structural properties.

A generic approach to analyse the possibility of active isolation, in a structural domain setting, was treated in Blanke and Staroswiecki (2006), and specific algorithms were provided in Gelso and Blanke (2009). The main idea is that in order to be structurally isolable, at least two different paths should exist in the structure graph from input to output or to residuals, in which a violated constraint participate in one path but not in the other. The input-output or input-residual behaviour will then be normal for the second path but not for the first.

Figure 1 shows that alternating paths: $u_{i}-a_{i}-F_{i}-c_{5}$ for $i=\left\{1, \ldots, N_{t}\right\}$, and $u_{i}-a_{i}-F_{i}-c_{5}-T_{j}-m_{8+j}-T_{m j}$ for $i=\left\{1, \ldots, N_{t}\right\}$ and $j=\left\{1, \ldots, N_{m}\right\}$ will give such paths.

The test signal applied can be a short harmonic sequence, long enough to enable certain discrimination from wave and wind disturbances in the signals.

Active isolation for linear systems was treated in Niemann (2006), who introduced a $\mathcal{H}_{\infty}$ setup for generic design and Poulsen and Niemann (2008), who analysed a CUSUM detection scheme in relation to active diagnosis.

\section{Controller design}

As described above, control actions could be fault accommodation or control reconfiguration. Before addressing the fault accommodation for mooring line faults, controller design in faultless conditions is first reviewed. 


\subsection{Controller design in faultless conditions}

Active control is performed by the thrusters of the vessel. The primary objective of a positioning control system is to keep the vessel in a fixed position, $\mathbf{p}_{\mathrm{d}}$, and heading angle, $\psi_{\mathrm{d}}$. In case of a PM system, the secondary objective is to keep the line tensions within a limited range to prevent line break. The second objective is usually achieved by the criterion that the distance between the desired position of the vessel and the field zero point, $\mathbf{p}_{0}$, is less than a critical value. The objectives are given as,

$$
O:\left\{\begin{array}{l}
\left|\psi-\psi_{\mathrm{d}}\right|<\psi_{\mathrm{w}}, \\
\left|\mathbf{p}-\mathbf{p}_{\mathrm{d}}\right|<p_{\mathrm{w}}, \\
\left|\mathbf{p}_{\mathrm{d}}-\mathbf{p}_{0}\right|<p_{\text {crit }} .
\end{array}\right.
$$

For PM system, the following definitions are made for convenience. A field zero point is defined as the position of the moored vessel where there is no environmental load acting on the vessel. An equilibrium position is defined as the position where the mean environmental loads acting on the vessel are balanced by the mooring forces.

The surge and sway control and heading control are usually done by an output-PID control law, according to

$$
\begin{aligned}
\boldsymbol{\tau}^{x y}= & -\mathbf{K}_{\mathrm{p}}^{x y} \mathbf{R}_{n b}(\psi) \tilde{\mathbf{p}}-\mathbf{K}_{\mathrm{i}}^{x y} \mathbf{R}_{b n}(\psi) \int_{0}^{t} \tilde{\mathbf{p}} \mathrm{d} t \\
& -\mathbf{K}_{\mathrm{d}}^{x y} \tilde{\boldsymbol{\nu}} \\
\tau_{\mathrm{c}}^{\psi}= & -K_{\mathrm{p}}^{\psi} \tilde{\psi}-K_{\mathrm{i}}^{\psi} \int_{0}^{t} \tilde{\psi} \mathrm{d} t-K_{\mathrm{d}}^{\psi} \tilde{\dot{\psi}},
\end{aligned}
$$

where $\tilde{\mathbf{p}}=\hat{\mathbf{p}}-\mathbf{p}_{\mathrm{d}} ; \tilde{\boldsymbol{\nu}}=\hat{\boldsymbol{\nu}}-\boldsymbol{\nu}_{\mathrm{d}} ; \mathbf{K}_{\mathrm{p}}^{x y}, \mathbf{K}_{\mathrm{i}}^{x y}$, and $\mathbf{K}_{\mathrm{d}}^{x y}$ are the non-negative $\mathrm{P}$, I, and D controller gain matrices; $\tilde{\psi}=\hat{\psi}-\psi_{\mathrm{d}} ; \tilde{\dot{\psi}}=\hat{\dot{\psi}}-r_{\mathrm{d}} ; \psi_{\mathrm{d}}$ and $r_{\mathrm{d}}$ are the desired heading and yaw rate, respectively; and $K_{\mathrm{p}}^{\psi}, K_{\mathrm{i}}^{\psi}$, and $K_{\mathrm{d}}^{\psi}$ are the nonnegative $\mathrm{P}, \mathrm{I}$, and $\mathrm{D}$ controller gains. The states with the hat $\left(^{\wedge}\right)$ are estimations from an observer, not discussed in this paper, which is used to filter the waveinduced motion and estimate velocity from the measured position. More details on design for positioning control can be found in Sørensen et al. (1996) and for position mooring control and observer design in Nguyen and Sørensen (2009a).

\subsection{Control architecture to obtain fault-tolerant Position-mooring}

A fault-tolerant control architecture for the PM system requires a control architecture that is implemented as shown in Fig. 4. In the Figure, solid purple lines indicate signals used in the closed loop control. Solid red lines show signals that are sent to residual generator and evaluation in the change detection function block. Signals on solid lines are transmitted with the sampling frequency of the control system. Dashed lines indicate signals that are event driven, i.e are sent when a fault is evaluated and a change needs to be made in either of the function blocks that execute the real time control. The function blocks are: 
Controller Input: position, heading, setpoints for position and heading, estimated velocities and turn rate. Calculates desired thrust and moment vectors and makes thruster allocation. Output: thrust commands.

Thrust allocation Comprises and updated thrust configuration matrix for the vessel and calculates the thrust demand from individual thrusters to obtain the desired X, Y forces and yaw moment N Fossen and Johansen (2013). The thrust configuration matrix reflects which thrusters are declared healthy by the hypothesis test function.

Observer Estimates velocities and turn rate. Filters first order wave effects from signals to controller. Observer instance will change according to which signals are declared healthy.

Sensors select Selects the set of healthy sensor signals that are passed on to the relevant observer.

Setpoint generator Calculates the desired position $\mathbf{p}_{d}$. Position reference can be changed if breakage of mooring lines is at risk.

Residual generator Calculates residual vector. The generation of residuals changes when the set of healthy components / signals change.

Change detector Performs a hypothesis test about which components and signals can be considered healthy.

FTC supervisor This function block keeps an account of the state of sensors and actuators, and of system parameters. It comprises computational logics and algorithms to determine which remedial actions are need to handle specific defects in the system. It signals an abstract system description to the remedial actions block, which implements the necessary actions in lower level software. In short, it assesses which components are healthy. Using this information, it ensures that only actuators, other components and signals are used that have been declared healthy. It avoids control schemes that use components or signals for which failure / faults would not be detectable.

Remedial actions function block Fault handling is performed. Mooring line failure typically by change of setpoint Fang and Blanke (2011); Thruster failure or glitch is typically handled in the thrust allocation calculation; Sensor faults are typically handled by estimating missing signals.

The generic architecture shown in Figure 4 is commonly applied for marine systems fault-tolerant sensor fusion and fault-tolerant control. Virtual Sensor or Virtual Actuator approaches could be alternatives to sensor and actuator fault handling when the required assumptions for the virtual approach holds Richter et al. (2011), Seron et al. (2013). 


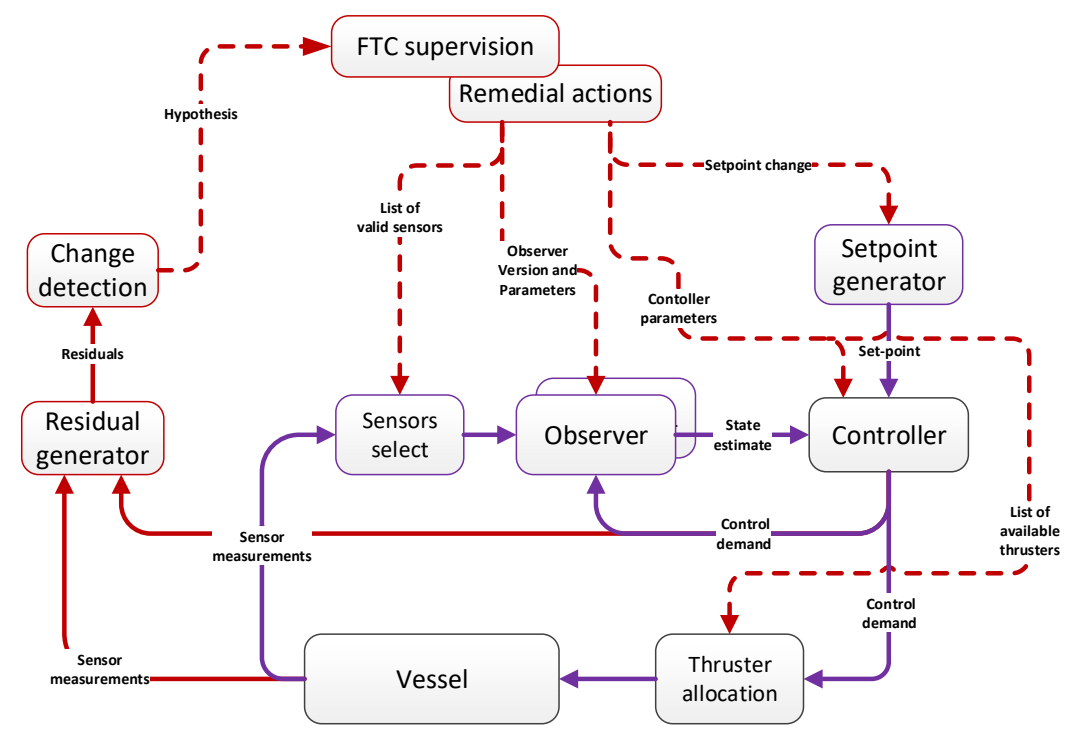

Figure 4: Control diagram of fault-tolerant positioning mooring control. 


\subsection{Faults in mooring lines}

We assume that in faultless conditions the thruster assistance only keeps the vessel's heading and adds damping in surge and sway, according to,

$$
\begin{aligned}
\boldsymbol{\tau}^{x y} & =-\mathbf{K}_{\mathrm{d}}^{x y} \tilde{\boldsymbol{\nu}} \\
\tau_{\mathrm{c}}^{\psi} & =-K_{\mathrm{p}}^{\psi} \tilde{\psi}-K_{\mathrm{i}}^{\psi} \int_{0}^{t} \tilde{\psi} \mathrm{d} t-K_{\mathrm{d}}^{\psi} \tilde{\dot{\psi}},
\end{aligned}
$$

When faults as line breakage or wrong pretension occur in a mooring line, the vessel will have another equilibrium position, and a minimum risk $\mathbf{p}_{d}$ can be calculated, see Fang et al. (2015). If the vessel's drift is small, the controller in Eqs. (31)-(32) should be redesigned with the updated plant by considering the updated mooring loads in the vessel's dynamics in Eq. (4). If the vessel's drift is large, the vessel needs to be kept in the position as in the faultless conditions. Necessary control forces may be obtained by calculating adequate feed forward during transient conditions following diagnosis of a mooring line breakage.

\subsection{Faults in sensors and sensor systems}

Physical faults in sensors and in inertial measurement units include fluctuation / jumps in signals, slow drift, bias, frozen signal or temporal unavailability. A jump in a measurement signal is rather easy to detect whereas incipient faults are more difficult. A strain gauge which is used to measure the tension in a mooring line often experiences a permanent drift after some time in service. Positioning devices may experience jumps and random drift for various reasons. For GNSS (global navigation satellite system) receivers when clock updates are made to satellites and when satellites in view change. Hydrophone position readings are influenced by temperature and salinity profiles in the water. Inertial measurement units suffer from time-varying bias in accelerometer and turn rate readings.

With the control system using the measurements for real time feedback, consequences of sensor faults can be serious. Therefore, the safe reaction to a device being declared faulty by the change detection is to disable the device suspected to be faulty. If there is physical redundancy of the devices, healthy devices are used instead. Without physical redundancy, the model based observer estimates the missing measurements.

\subsection{Faults in thrusters}

Faults in thrusters usually include temporal loss of power, failure to zero, failure to full, shaft speed freeze or reduced thrust generation due to sea weed. An azimuth thruster may experience fixed angle or loss of hydraulic pressure causing frozen azimuth or slow rotation.

If a thruster fails to follow a commanded thrust, it must be disabled and thrust allocation must be redesigned for the healthy thrusters. If an azimuth thruster fails to stay at the desired angle, the thrust allocation is redesigned 
with the consideration of this fixed angle. Such fault tolerant control actions are part of the system reconfiguration.

The advantage of the approach we use is that we do not need to specify the physical nature of particular faults. The methodology detects deviation of normal behaviour of components. Therefore, the remedial action will be to disregard a faulty component from a control solution when doing the reconfiguration needed to handle a failure.

\subsection{Role of single input-output sanity check}

In any automated system, the first row of defence against failures is always sanity check of input and output signals. The standard approaches include to have supervised input-output to protect against cable failures, to have double supervised digital switches signalling safety related binary information, to have watchdog software supervising that local area network transmission of signals is alive, etc. These sanity check types of supervision of single input-output are well documented in standards to meet functional safety requirements, which are prerequisites to obtain equipment approval by classification societies. Common source failures, i.e. loss of power to sensors and or actuators, are detected by similar means. Absence of live signal feedback from sensors are hence always detected as failures in automated systems. The treatment in this paper deals with the more subtle failures, where analytical redundancy checks are needed to isolate the faulty component(s).

\section{Validation}

\subsection{Overviews}

An FTC structure for positioning control systems is proposed in Fig. 4. All the signals from sensors and measurement units are checked before entering the fault detection block. If a fault is detected, the supervisor will take appropriate actions in sensors and measurement units, controlled plant or reallocation of thrusters to handle this fault.

The purpose of this Section is to validate the FTC designs by comparing the performances of the vessel with and without FTC when faults occur. Both simulations and experiments are used for validation. The simulation was carried out with a turret moored FPSO operating in 380-meter depth at Norwegian Sea. The FPSO has a mass, $m=166 \times 10^{3}$ tons, length, $L=271 \mathrm{~m}$, breadth, $B=41 \mathrm{~m}$ and draft, $D=15.5 \mathrm{~m}$. The turret mooring system consists of twelve lines $\left(N_{\mathrm{m}}=12\right.$, see Fig. 6$)$ each of which has three segments. The parameters of the mooring lines are presented in Table 10. The simulations was carried out using the Marine System Simulator (MSS) developed in NTNU.

The experiments were carried out using the model vessel, Cybership III (Fig. 7), which is a $1: 120$ scale model of the FPSO of the simulation, having a mass, $m=75 \mathrm{~kg}$, length, $L=2.27 \mathrm{~m}$, and breadth, $B=0.4 \mathrm{~m}$. The turret mooring system consists of four lines (Fig. 6). The vessel is equipped with two main azimuth propellers, one tunnel thruster and one front azimuth thruster. 


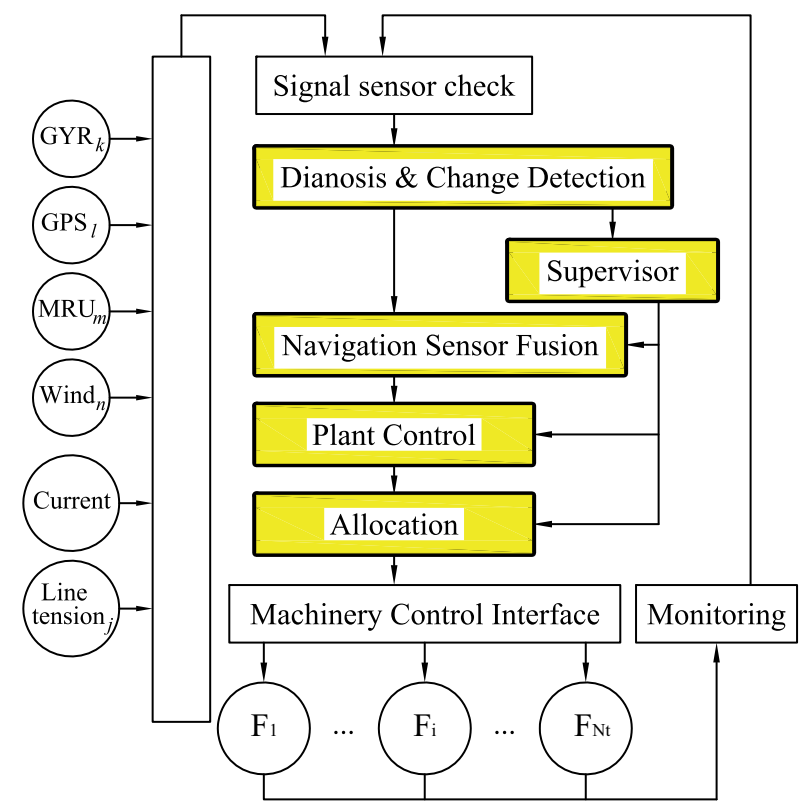

Figure 5: Structure of fault-tolerant control software for positioning control.

The internal hardware architecture is controlled by an onboard computer which can communicate with an onshore PC through a WLAN. An onshore 4-camera measurement system provides Earth-fixed position and heading. A wave maker system was used to simulate JONSWAP-distributed waves. The experiments were performed in the Marine Cybernetics Laboratory (MCLab) at NTNU. The experimental results presented are converted into full scale. In the experiments, a pulley system was used to simulate the effects of mean loads due to wind and current, as illustrated in Fig. 7.

In the simulations and experiments, the environmental load direction was collinear and $15^{\circ}$ relatively to the bow of the vessel (Fig. 6). The simulation and experiment were performed with a significant wave height, $H_{\mathrm{s}}=10 \mathrm{~m}$, wave period, $T_{\mathrm{p}}=14.18 \mathrm{~s}$ (JONSWAP distributed wave), wind velocity, $v_{10}=$ $22.41 \mathrm{~m} / \mathrm{s}$, and current velocity, $v_{\mathrm{c}}=0.5 \mathrm{~m} / \mathrm{s}$.

The following subsections will present the simulation and experimental results for the cases of real faults. We considered the single fault scenarios with step and slowly-varying additive faults, i.e. a mooring line break and a measurement drift in a position measurement unit, and with failure to zero fault in a thruster. A multi fault scenario was also considered with two simultaneous faults, one which is a wrong pretension in a mooring line and another, occurring later, is a jump in GPS position measurement. 


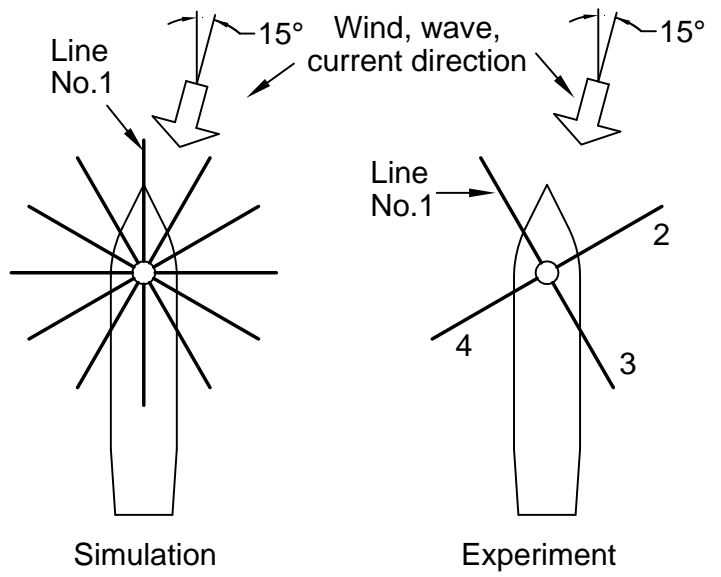

Figure 6: Mooring systems and drection of environmental loads.

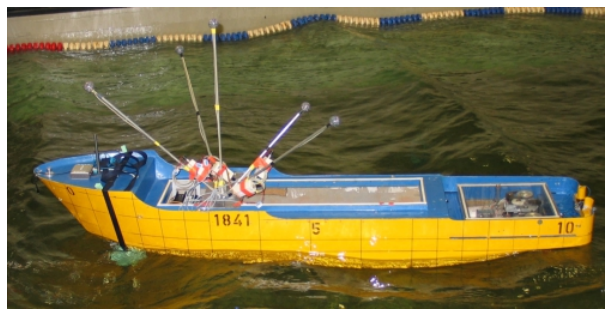

(a) CS3

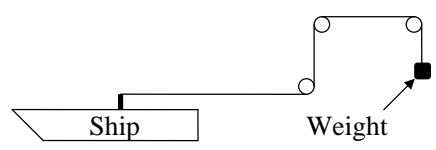

(b) Loads

Figure 7: The Cybership III (left) and the pulley system to simulate mean wind and current loads (right). 
Table 10: Parameters of the mooring lines.

\begin{tabular}{lccc}
\hline & $\begin{array}{c}\text { Segment 1 } \\
\text { (near turret) }\end{array}$ & Segment 2 & Segment 3 \\
\hline$E$ modulus $\left(10^{8} \mathrm{~N} / \mathrm{m}^{2}\right)$ & 838.5 & 1126 & 979.7 \\
Unstretched length, $L(\mathrm{~m})$ & 1060 & 380 & 80 \\
Diameter, $D(\mathrm{~m})$ & 0.137 & 0.121 & 0.114 \\
Cable density, $\rho_{c}(\mathrm{~kg} / \mathrm{m})$ & 1178 & 1265 & 1178 \\
Added mass coef., $C_{\mathrm{mn}}$ & 1.5 & 1.5 & 1.5 \\
Normal drag coef., $C_{\mathrm{dn}}$ & 2.5 & 2.5 & 2.5 \\
\hline
\end{tabular}

\subsection{Line breakage}

The vessel was first operated with a faultless mooring system and then with a line breakage occurring in the mooring line 1 (Fig. 6). We will, in this subsection, show only the experimental results since the simulation results are similar.

Fig. 8 shows North-East position of the vessel and parity relation $r_{1}$ with the corresponding fault detection signal. The figure shows that when line 1 broke, the mean of the residual $r_{1}$ changed. When the fault occurred, the driftoff of the vessel without FTC was to the South causing large tensions in the mooring lines 2 and 4 (Fig. 9). We observe that the vessel with FTC performed similarly to the faultless scenario meaning that the vessel's drift was reduced and the tensions in mooring lines were maintained within a normal range (Figs. 8 and 9). The FTC in this experiments is mooring line fault accommodation presented in Section 5.3.

\subsection{Wrong pretension and position measurement jump}

This subsection will show the experimental results for two simultaneous faults. The vessel was first operated in faultless conditions. After a while line 1 was loosened to simulate a wrong pretention and then a sudden jump in position measurement for a short duration of time. Figures 10 and 11 show the vessel's position and the tensions in mooring lines with and without FTC. The results show that the effect of FTC for wrong pretension was similar to that for line breakage. The effect of the subsequent jump in position measurement would be further drift of the vessel in addition to the drift due to loosening in line 1, when there was no FTC. The FTC handled the jump in position measurement by reconfigurating the control system such that the position prediction from the observer replaced the faulty measurement. Consequently, the vessel was still kept in the position and the tensions of the mooring lines were in a normal range. 

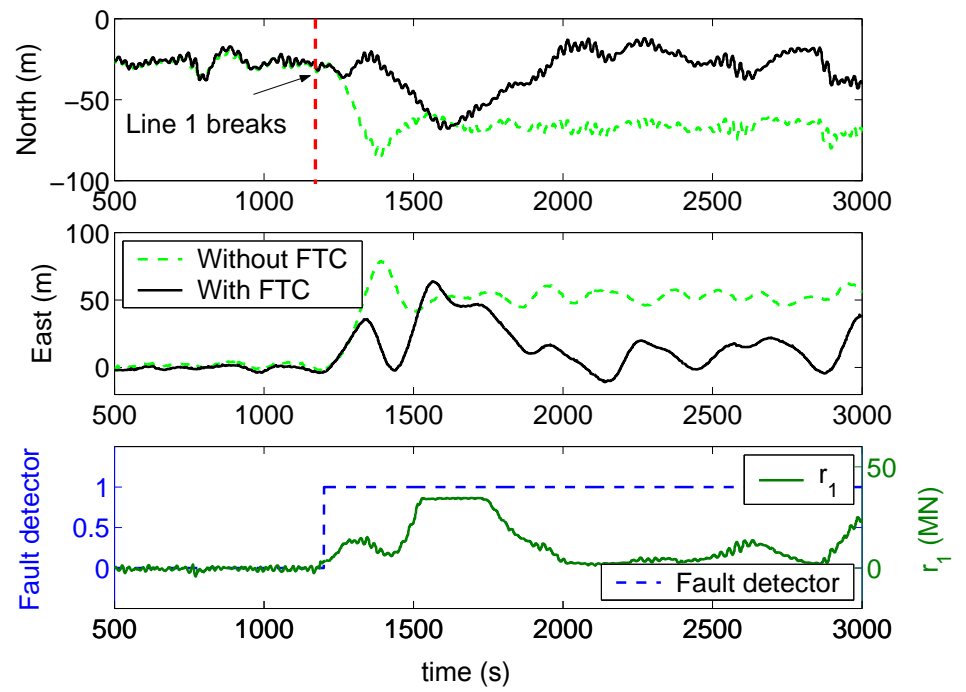

Figure 8: North-East position of the vessel and parity relation $r_{1}$ and fault detection signal.
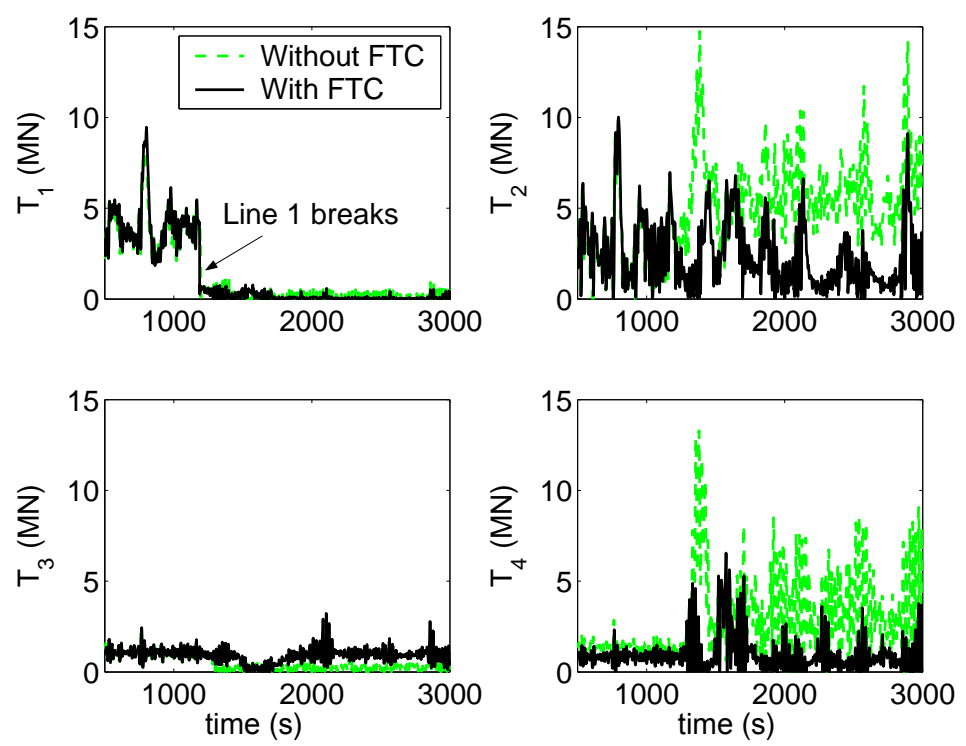

Figure 9: Tensions of mooring lines. 

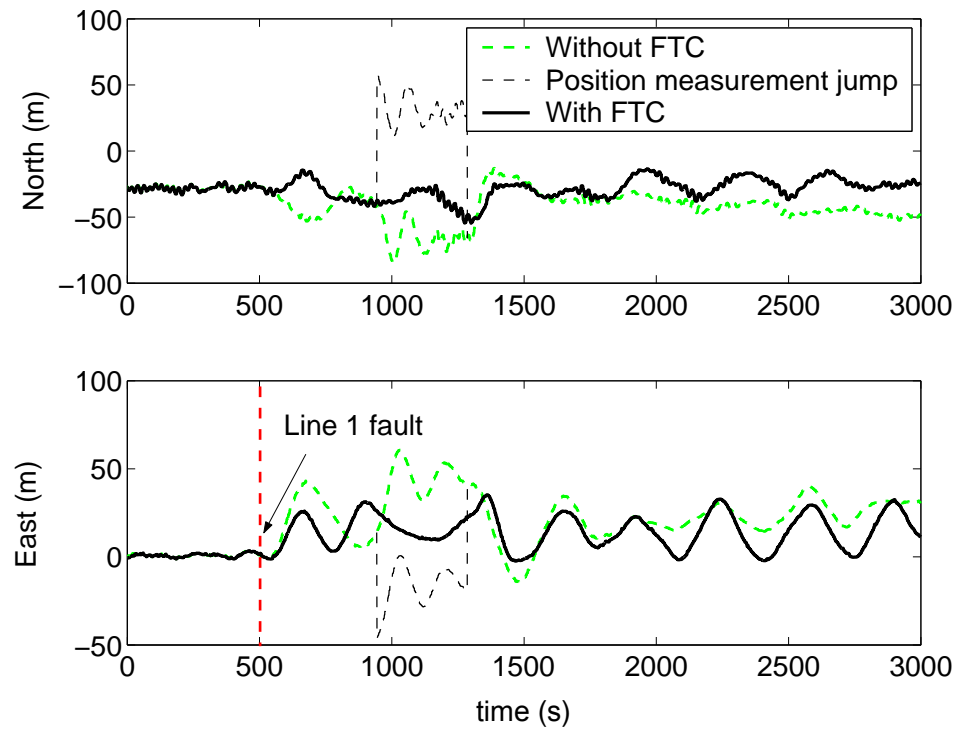

Figure 10: Vessel's position of vessel subjected to wrong pretension and later a jump in position measurement.
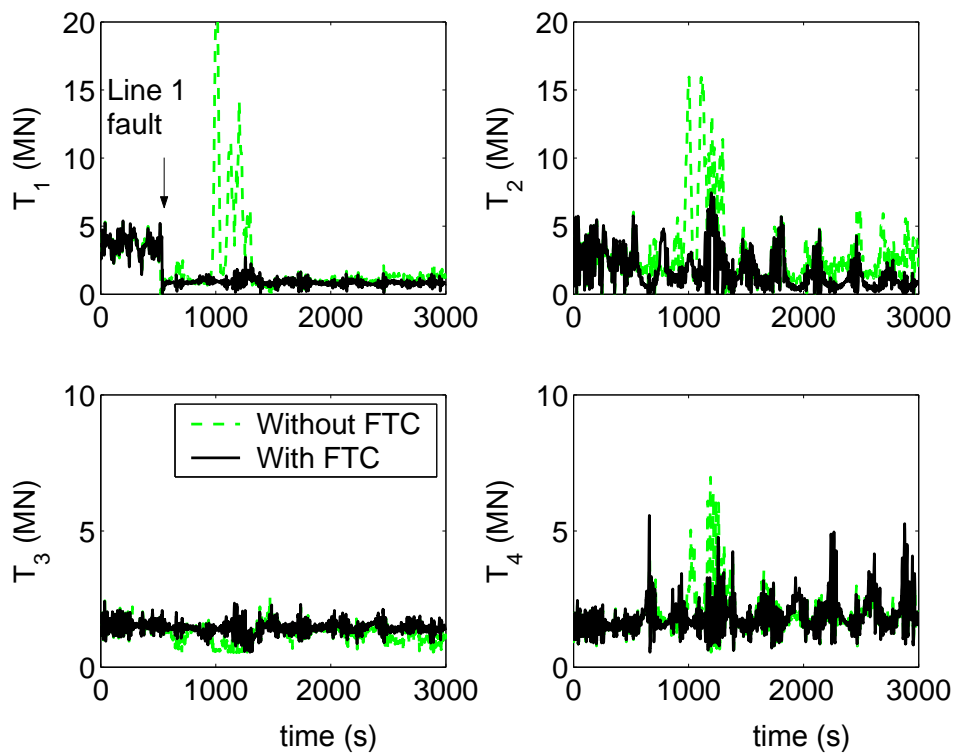

Figure 11: Tensions subjected to wrong pretension and later a jump in position measurement. 

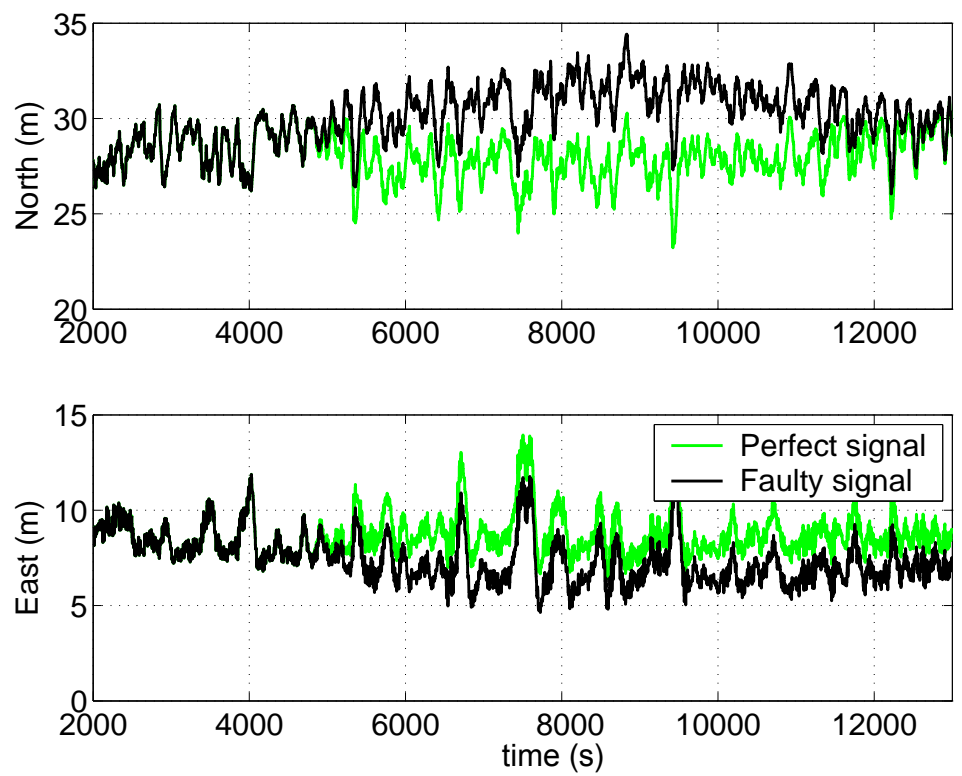

Figure 12: Perfect and faulty signals of GPS receiver 1.

\subsection{Slow drift in position measurement}

Firstly, we performed an experiment with two GNSS (GPS) receivers which were fixed on the ground by recording the measurements from the two receivers. With receivers' positions known, we can calculate the deviations in the measurement data. Secondly, these deviations after properly scaled were used as perturbations to the perfect signals of two virtual GPS receivers on the Cybership III performing a DP operation with AUT class. The perfect signals of the virtual GPS receivers were calculated from the vessel position obtained by the four-camera system (see Section 6.1) and the virtual locations of the receivers on the vessel. The vessel was first operated in the condition of perfect position measurements. After a while, the measured position for feedback was the perfect position perturbed by the deviations from the real GPS receivers. Figs. 12 and 13 show the perfect and faulty position measurements of the two virtual GPS receivers on the vessel without FTC. It is observed that the quality of the GPS receiver 2 was better than that of the receiver 1.

Fig. 14 shows the position of the vessel with and without FTC. We observe that the vessel's drift without FTC was approximately $1 \mathrm{~m}$ to South and $1 \mathrm{~m}$ to East while that with FTC was almost unnoticeable. This is explained by the fact that the FTC detected slow drift in the signal from the receiver 1; consequently FTC disabled the GPS receiver 1 and used the receiver 2 for feedback. 

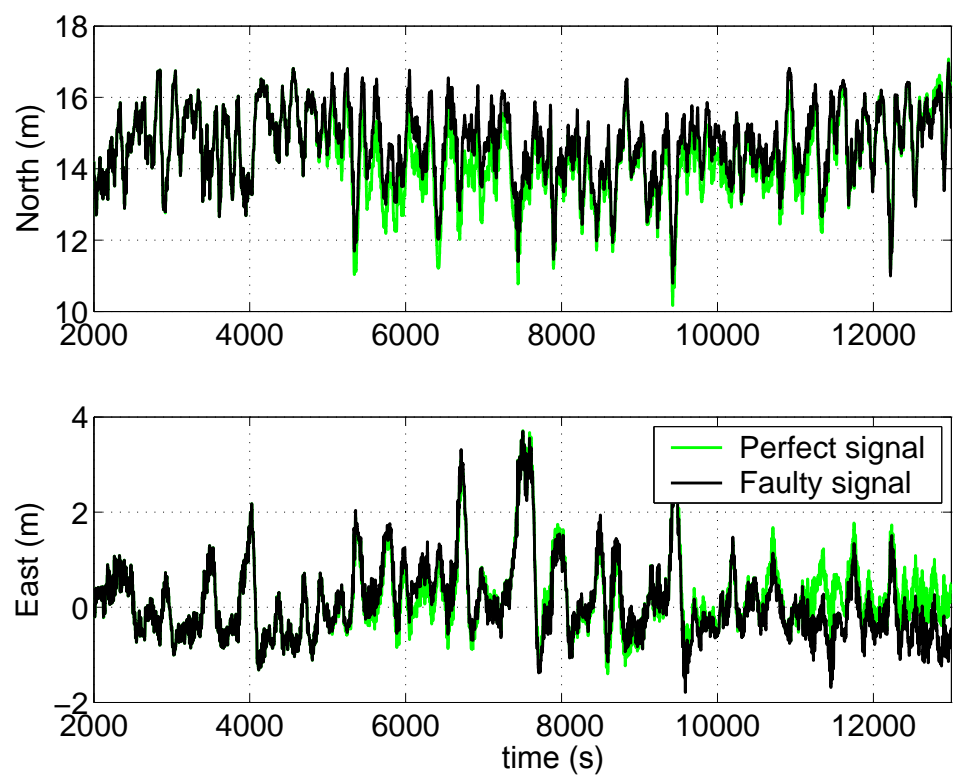

Figure 13: Perfect and faulty signals of GPS receiver 2.
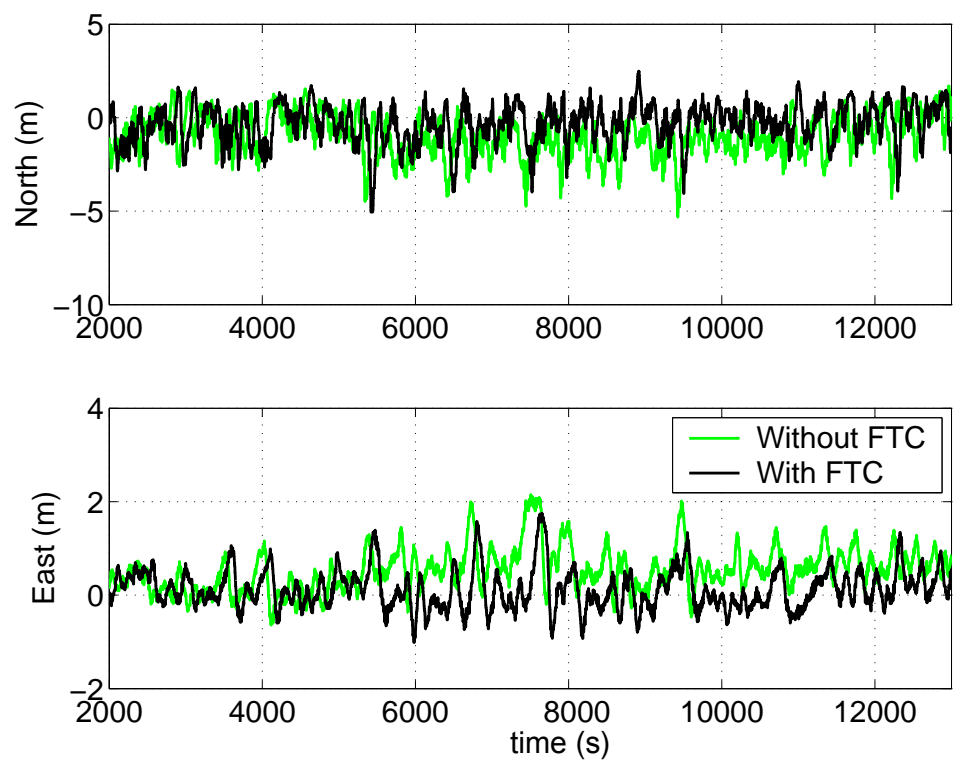

Figure 14: Vessel's position when the signals from GPS receivers is subjected to slowly-varying additive faults. 
Table 11: Active isolation dependency matrix.

\begin{tabular}{ccccc}
\hline & $T_{\mathrm{m}_{1}}$ & $T_{\mathrm{m}_{2}}$ & $T_{\mathrm{m}_{3}}$ & $T_{\mathrm{m}_{4}}$ \\
\hline$u_{1}$ & $x_{11}$ & $x_{12}$ & $x_{13}$ & $x_{14}$ \\
$u_{2}$ & $x_{21}$ & $x_{22}$ & $x_{23}$ & $x_{24}$ \\
$u_{3}$ & $x_{31}$ & $x_{32}$ & $x_{33}$ & $x_{34}$ \\
$u_{4}$ & $x_{41}$ & $x_{42}$ & $x_{43}$ & $x_{44}$ \\
\hline
\end{tabular}

\subsection{Thruster failures}

From the structural analysis with the assumption of disregarding fault detector signal from the thruster, faults in thrusters are only structurally detectable and not structurally isolable. The active isolation can be used to deal with these detectable faults. In this technique, we will perturb the system with a sinusoidal signal from a thruster. From the structure graph (Fig. 1), we know that tensions in all mooring lines will be affected if a perturbation signal is added to a normal thrust demanded by the positioning control system. The amplitudes of the tension responses at the frequency of the sinusoidal perturbations are estimated for faultless conditions in advance. If the online estimations are not as those in faultless conditions, the fault can be isolated based on a so-called active isolation dependency matrix Blanke and Staroswiecki (2006), which structurally maps the thrust inputs, $u_{i}$, to the tension outputs, $T_{\mathrm{m}_{j}}$. Such matrix for a PM vessel with four mooring lines and four thrusters are shown in Table 11. The element of this matrix is $x_{i j}$ which reads $x_{i j}=0$ if an online estimation of a tension is similar to that in faultless conditions and reads $x_{i j}=1$ if not. If a row of the matrix is one then the corresponding thruster is faulty. If a column of the matrix is one then the corresponding tension measurement unit is faulty.

Simulations with a 'failure to zero' in thruster 1 is shown to validate the active isolation and to demonstrate the thrust reallocation. There are three cases in the simulations, a healthy and two faulty cases. The simulations and model tests include cases with and without active isolation. The active isolation was activated when a fault was detected but could not be isolated by the passive diagnosis approach. The perturbations used for active diagnosis are here sinusoidal signals. The dependency matrix was determined (see Table 12). The active isolation dependency matrix shows that the fault was in thruster 1.

Figs. 15 and 16 show the vessel's position and mooring line tensions in no-fault condition and then in 'failure to zero' fault in thruster 1 . For the faulty condition cases, it was observed that the performance of the system with FTC was not improved right after the occurrence of the fault compared to that without FTC. This is due to the fact that the active isolation took some time to actively diagnose the fault in thruster 1 . Once the fault was isolated, the FTC switched to the allocation with three thrusters. Consequently, the performance of the PM vessel was back to normal in terms of position and tensions. 
Table 12: Active isolation dependency matrix for simulation.

\begin{tabular}{ccccc}
\hline & $T_{\mathrm{m}_{1}}$ & $T_{\mathrm{m}_{2}}$ & $T_{\mathrm{m}_{3}}$ & $T_{\mathrm{m}_{4}}$ \\
\hline$u_{1}$ & $x_{11}=1$ & $x_{12}=1$ & $x_{13}=1$ & $x_{14}=1$ \\
$u_{2}$ & $x_{21}=0$ & $x_{22}=0$ & $x_{23}=0$ & $x_{24}=0$ \\
$u_{3}$ & $x_{31}=0$ & $x_{32}=0$ & $x_{33}=0$ & $x_{34}=0$ \\
$u_{4}$ & $x_{41}=0$ & $x_{42}=0$ & $x_{43}=0$ & $x_{44}=0$ \\
\hline
\end{tabular}
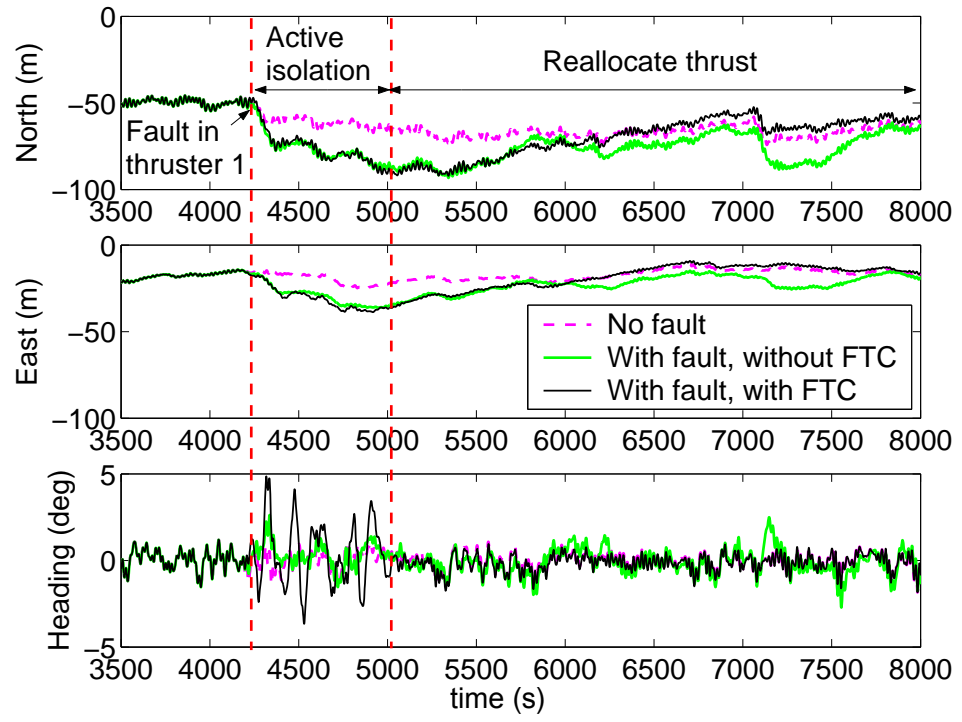

Figure 15: Vessel's position subjected to failure to zero fault in thruster 1. 

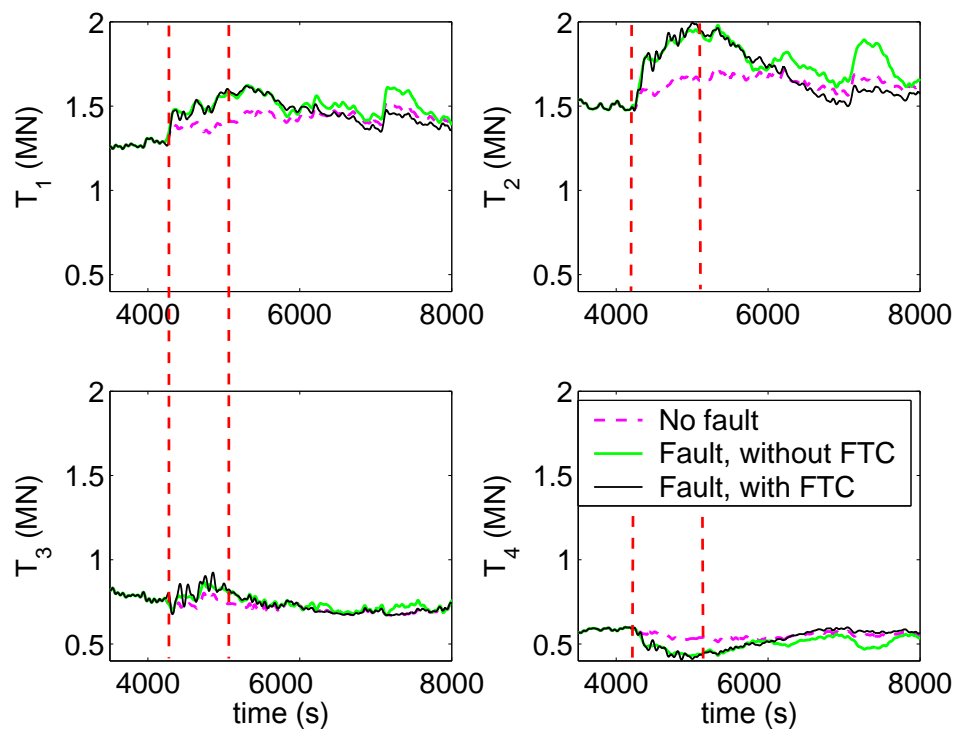

Figure 16: Mooring line tensions subjected to failure to zero fault in thruster 1.

\section{Concluding remarks}

This paper addressed fault-tolerant control for positioning control systems of vessels in general and Position-moored vessels in particular. A methodology was presented that allowed assessment of safe Position-mooring control under single and multiple faults. Fault diagnosis was designed through structure graph analysis of a model of a vessel expressing overall normal behaviours. Analysis of residuals showed that several faults, including mooring line breakage or mooring line tension sensor failure, were only detectable, whereas isolation is required to make the control system take the correct remedial actions to faults.

Active isolation of faults was introduced to alleviate this problem. Statistical change detection was applied to determine when a fault had happened. Time to detect and time between false alarm were used as design criteria for change detection design in the presence of significant wave disturbances in the signals. Fault accommodation and system reconfiguration methods were developed for the different types of faults and control actions to handle faults were demonstrated by model basin tests for selected faults with high severity.

Simulations and experiments were carried and multiple faults in mooring lines, position measurement units and thrusters, and showed that FTC could improve the performance and increase the safety of the vessel in the faulty conditions.

The topic presented in this paper is essential for the design of autonomous vessels since the principles presented are fundamental to achieve fault-tolerant behaviours. Analysis of overall safety and analysis of risk related to such designs 
will be interesting topics of further research.

\section{Acknowledgement}

The support of this research from the Research Council of Norway through Centres of Excellence Programme (grants 146025-CeSOS and 223254-AMOS), are gratefully acknowledged. Mr. Torgeir Wahl is gratefully acknowledged for his support during the model tank experiments.

The authors would like to thank the anonymous reviewers for very detailed and useful suggestions for improving the paper.

\section{References}

Aamo, O.M., Fossen, T.I., 2001. Finite element modelling of moored vessels. Mathematical and Computer Modelling of Dynamical Systems , 47-75.

Balchen, J.G., Jenssen, N.A., Mathisen, E., Sælid, S., 1980. A dynamic positioning system based on kalman filtering and optimal control. Modeling, Identification and Control 1, 135-163.

Basseville, M., Nikiforov, I., 1993. Detection of Abrupt Changes: Theory and Application. Prentice Hall, New York.

Basseville, M., Nikiforov, I., 2002. Fault isolation for diagnosis: Nuisance rejection and multiple hypothesestesting. Annual Reviews in Control 26.

Benetazzo, F., Ippoliti, G., Longhi, S., Raspa, P., 2015. Advanced control for fault-tolerant dynamic positioning of an offshore supply vessel. Ocean Engineering 106, 472-484. Cited By 3.

Berntsen, P., Aamo, O., Leira, B., 2008. Structural reliability-based control of moored interconnected structures. Control Engineering Practice, 495-504.

Besançon, G., 2003. High-gain observation with disturbance attenuation and application to robust fault detection. Automatica 39, 1195-1102.

Blanke, M., 2005. Diagnosis and fault-tolerant control for ship station keeping, in: IEEE International Symposium on Intelligent Control and 13th Mediterranean Conference on Control and Automation, Cyprus. pp. 1385-1390.

Blanke, M., 2006. Fault-tolerant sensor fusion for marine navigation, in: Proceedings of the 7th IFAC Conference on Marine Crft Manoeuvring and Control (MCMC'2006), Lisbon, Portugal. pp. 1385-1390.

Blanke, M., Fang, S., Galeazzi, R., Leira, B.J., 2012. Statistical change detection for diagnosis of buoyancy element defects on moored floating vessels. IFACPapersOnLine 45. 
Blanke, M., Izadi-Zamanabadi, R., Lootsma, T., 1998. Fault monitoring and reconfigurable control for a ship propulsion plant. Journal of Adaptive Control and Signal Processing 12, 671-688.

Blanke, M., Kinnaert, M., Lunze, J., Staroswiecki, M., 2015. Diagnosis and Fault-Tolerant Control. Springer. 3rd edition.

Blanke, M., Lorentzen, T., 2006. Satool - a tool for structural analysis of complex automation systems, in: 14th IFAC Safeprocess'2006, Beijing. pp. 673-678.

Blanke, M., Staroswiecki, M., 2006. Structural design of systems with safe behavior under single and multiple faults, in: 6th IFAC Symposium on Fault Detection, Supervision and Safety of Technical Processes SAFEPROCESS, Beijing, P. R. China. pp. 511-516.

Breivik, M., Kvaal, S., Østby, P., 2015. From eureka to k-pos: Dynamic positioning as a highly successful and important marine control technology. IFACPapersOnLine 48 (16), 313-323.

Chen, H., Moan, T., 2004. Probabilistic modeling and evaluation of collision between shuttle tanker and fpso in tandem offloading. Reliability Engineering \& System Safety 84, 169-186.

Chen, H., Moan, T., Verhoeven, H., 2009. Effect of dgps failures on dynamic positioning of mobile drilling units in the north sea. Accident Analysis and Prevention 41 (6), 1164 - 1171.

DNV, 2014. Dynamic positioning systems. Rules for Classification of Ships , Part 6 Chapter 7 .

Dulmage, A.L., Mendelsohn, N.S., 1959. A structure theory of bi-partite graphs of finite exterior dimension. Trans. Royal Society of Canada. Ser III. 53, 1-13.

Edwards, C., Spurgeon, S.K., 2000. A sliding mode observer based fdi scheme for the ship benchmark. European Journal of Control 6, 341 .

Fang, S., Blanke, M., 2011. Fault monitoring and fault recovery control for position-moored vessels. Int. J. of Applied Computer Science and Control (AMCS) 21 (3).

Fang, S., Blanke, M., Leira, B., 2013. Position mooring control based on a structural reliability criterion. Structural Safety 41, $97-106$.

Fang, S., Blanke, M., Leira, B.J., 2015. Mooring system diagnosis and structural reliability control for position moored vessels. Control Engineering Practice $36,12-26$.

Feng., Z., Liang, M., Chu, F., 2013. Recent advances in time-frequency analysis methods for machinery fault diagnosis: A review with application examples. Mechanical Systems and Signal Processing 38, 165-205. Cited By (since 1996):15. 
Fossen, T.I., 2002. Marine Control Systems: Guidance Navigation and Control of Ships Rigs and Underwater Vehicles. Marine Cybernetics, Trondheim, Norway.

Fossen, T.I., Johansen, T.A., 2013. Control allocation - a survey. Automatica 49, 1087-1103.

Garcia, E.A., Frank, P.M., 1997. Deterministic nonlinear observer-based apapproach to fault diagnosis: a survey. Control Engineering Practice 5, 663-670.

Gelso, E., Blanke, M., 2009. Structural analysis extended with active fault isolation - methods and algorithms, in: Proceedings 7th IFAC SAFEPROCESS'2009 Symposium, pp. 597-602.

Gray, J.N.P., Macdonald, I.F., 1982. Safety study of part of a dynamic positioning system for a diving-support ship. Reliability Engineering 3, 179-192.

Hassani, V., Srensen, A., Pascoal, A., Athans, M., 2017. Robust dynamic positioning of offshore vessels using mixed-? synthesis modeling, design, and practice. Ocean Engineering 129, 389-400. Cited By 0.

IMO, 1994. Guidelines for vessels with dynamic positioning systems. MSC/Circ.645. International Maritime Organization, London, England.

Izadi-Zamanabadi, R., Blanke, M., 1999. A ship propulsion system as a benchmark for fault-tolerant control. Control Engineering Practice 7, 227-239.

Kay, S., 1998. Fundamentals of Statistical Signal Processing, Volume 2: Detection Theory. Prentice Hall.

Krysander, M., 2006. Design and Analysis of Diagnosis Systems Using Structural Methods. Ph.D. thesis. Linköping University.

Laursen, M., Blanke, M., Düştegör, D., 2008. Fault diagnosis in a water for injection system using enhanced structural isolation. International Journal of Applied Mathematics and Computer Science 18 (4), 593-603.

Lindegaard, K.P.W., 2003. Acceleration feedback in Dynamic Positioning. Phd thesis. Department of Engineering Cybernetics, NTNU. Norway.

Nguyen, D., Sørensen, A., 2009a. Switched control for thruster-assisted position mooring. Control Engineering Practice, 985-994.

Nguyen, T.D., Sørensen, A.J., 2009b. Setpoint chasing for thruster-assisted position mooring. Ocean Engineering 34, 548-558.

Nguyen, T.D., Sørensen, A.J., Quek, S.T., 2007. Design of high level hybrid controller for dynamic positioning from calm to extreme sea conditions. $\mathrm{Au}-$ tomatica $43,768-785$. 
Niemann, H., 2006. A setup for active fault diagnosis. IEEE Trans. on Automatic Control 51, 1572-1578.

Niemann, H., 2012. Sl titlen op. AMCS .

Noura, H., Theilliol, D., Ponsart, J.C., Cham, A., 2009. Fault-tolerant Control Systems - design and Practical Applications. Springer.

Park, K.P., Jo, A.R., Choi, J.W., 2016. A study on the key performance indicator of the dynamic positioning system. International Journal of Naval Architecture and Ocean Engineering 8, 511-518. Cited By 0.

Persis, C.D., Isidori, A., 2001. A geometric approach to nonlinear fault detection and isolation. IEEE Transactions of Automatic Control 46, 853-865.

Poulsen, N.K., Niemann, H., 2008. Active fault diagnosis based on stochastic tests. Int. J. of Applied Mathematics and Computer Science 18 (4), 487-496.

Ren, Z., Skjetne, R., 2016. A tension-based position estimation solution of a moored structure and its uncertain anchor positions, in: IFAC-PapersOnLine, Elsevier. pp. 251-257.

Richter, J., Heemels, W., Van De Wouw, N., Lunze, J., 2011. Reconfigurable control of piecewise affine systems with actuator and sensor faults: Stability and tracking. Automatica 47,678-691. Cited By 58.

Selkäinaho, J., 1993. Tuning a dynamic positioning system. Automatica 3, $865-875$.

Seron, M., De Don, J., Richter, J., 2013. Integrated sensor and actuator faulttolerant control. International Journal of Control 86, 689-708. Cited By 4 .

Sørensen, A.J., 2005. Structural issues in the design and operation of marine control systems. Annual Reviews in Control 29, 125-149.

Sørensen, A.J., Leira, B.J., Strand, J.P., Larsen, C.M., 2001. Optimal setpoint chasing in dynamic positioning of deep-water drilling and intervention vessels. International Journal of Robust and Nonlinear Control 11, 1187-1205.

Sørensen, A.J., Sagatun, S.I., Fossen, T.I., 1996. Design of a dynamic position system using model-based control. Control Engineering Practice 4, 359-368.

Sørensen, A.J., Strand, J.P., 2000. Positioning of small-waterplane-area marine constructions with roll and pitch damping. Control Engineering Practice 8, 205-213.

Staroswiecki, M., Declerck, P., 1989. Analytical redundancy in nonlinear interconnected systems by means of structural analysis, in: Proc. IFAC AIPAC'89 Symposium., Elsevier - IFAC. pp. 23-27. 
Strand, J.P., 1999. Nonlinear Position Control Systems Design for Marine Vessels. Phd thesis. Department of Engineering Cybernetics, NTNU. Norway.

Strand, J.P., Sørensen, A.J., Fossen, T.I., 1998. Modelling and control of thruster assisted position mooring system for ships. Modelling, Identification and Control 19, 65-71.

Sun, H., He, Z., Zi, Y., Yuan, J., Wang, X., Chen, J., He, S., 2014. Multiwavelet transform and its applications in mechanical fault diagnosis - a review. Mechanical Systems and Signal Processing 43, 1-24.

Svärd, C., Nyberg, M., Frisk, E., Krysander, M., 2013. Automotive engine FDI by application of an automated model-based and data-driven design methodology. Control Engineering Practice 4, 455-472.

Svärd, C., Nyberg, M., Frisk, E., Krysander, M., 2014. Data-driven and adaptive statistical residual evaluation for fault detection with an automotive application. Mechanical Systems and Signal Processing 45 (1), 170-192.

Tannuri, E., Morishita, H., 2006. Experimental and numerical evaluation of a typical dynamic positioning system. Applied Ocean Research 28, 133-146.

Travé-Massuyès, L., 2014. Bridging control and artificial intelligence theories for diagnosis: A survey. Engineering Applications of Artificial Intelligence 27, $1-16$.

Travé-Massuyès, L., Escobet, T., Olive, X., 2006. Diagnosability analysis based on component supported analytical redundancy relations. IEEE Trans. on Systems, Man and Cybernetics, Part A : Systems and Humans 36, 11461160.

Triantafyllou, M.S., 1990. Cable Mechanics with Marine Applications. Cambridge, MA 02139, Dept. of Ocean Engineering, Massachusetts Institute of Technology, USA.

Wang, L., Yang, J., He, H., Xu, S., Su, T.C., 2016. Numerical and experimental study on the influence of the set point on the operation of a thruster-assisted position mooring system. International Journal of Offshore and Polar Engineering 26, 423-432. Cited By 0.

Wang, Y., Zou, C., Ding, F., Dou, X., Ma, Y., Liu, Y., 2014. Structural reliability based dynamic positioning of turret-moored fpsos in extreme seas. Mathematical Problems in Engineering .

Willems, J.C., 1996. The behavioural approach to systems and control. European Journal of Control 2, 250-259.

Wu, D., Ren, F., Zhang, W., 2016. An energy optimal thrust allocation method for the marine dynamic positioning system based on adaptive hybrid artificial bee colony algorithm. Ocean Engineering 118, 216-226. Cited By 0. 
${ }_{974} \mathrm{Wu}$, N.E., Thavamani, S., Zhang, Y., Blanke, M., 2006. Sensor fault masking 975 of a ship propulsion system. Control Engineering Practice 14, 1337-1345. 